\title{
Quantum Instanton Evaluations of the Thermal Rate Constants for Complex Systems
}

\author{
Yi Zhao ${ }^{1}$ and Wenji Wang ${ }^{2}$ \\ ${ }^{1}$ State Key Laboratory for Physical Chemistry of Solid Surfaces and Fujian Provincial Key Lab of Theoretical and Computational \\ Chemistry, College of Chemistry and Chemical Engineering, Xiamen University, Xiamen 361005, China \\ ${ }^{2}$ College of Science, Northwest A\&F University, Yangling 712100, Shaanxi Province, China
}

Correspondence should be addressed to Yi Zhao, yizhao@xmu.edu.cn and Wenji Wang, wjwang@nwsuaf.edu.cn

Received 28 June 2011; Revised 26 August 2011; Accepted 10 September 2011

Academic Editor: Laimutis Bytautas

Copyright ( $) 2012$ Y. Zhao and W. Wang. This is an open access article distributed under the Creative Commons Attribution License, which permits unrestricted use, distribution, and reproduction in any medium, provided the original work is properly cited.

Quantum instanton (QI) approximation is recently proposed for the evaluations of the chemical reaction rate constants with use of full dimensional potential energy surfaces. Its strategy is to use the instanton mechanism and to approximate time-dependent quantum dynamics to the imaginary time propagation of the quantities of partition function. It thus incorporates the properties of the instanton idea and the quantum effect of partition function and can be applied to chemical reactions of complex systems. In this paper, we present the QI approach and its applications to several complex systems mainly done by us. The concrete systems include, (1) the reaction of $\mathrm{H}+\mathrm{CH}_{4} \rightarrow \mathrm{H}_{2}+\mathrm{CH}_{3}$, (2) the reaction of $\mathrm{H}+\mathrm{SiH}_{4} \rightarrow \mathrm{H}_{2}+\mathrm{SiH}_{3}$, (3) $\mathrm{H}$ diffusion on $\mathrm{Ni}(100)$ surface; and (4) surface-subsurface transport and interior migration for $\mathrm{H} / \mathrm{Ni}$. Available experimental and other theoretical data are also presented for the purpose of comparison.

\section{Introduction}

The accurate and efficient evaluation of chemical reaction rate constant is one of prime objectives of theoretical reaction dynamics. Since rigorous quantum mechanical approaches are limited to small molecular (several atoms) reactions, a variety of approximation approaches have been proposed. Benefited from the small recrossing dynamics at not-toohigh temperatures, the transition state theories (TSTs), originally proposed by Eyring [1,2] and Wigner [3], have become a possible and popular way to estimate rate constants. Due to their practical simplicity, they have been broadly applied to numerous reactions. The TST is inherently a classical theory and suitable at sufficiently high temperatures, where the classical description of nuclear motions may be adequate. At low temperatures, especially for the reactions involving the motions of light atoms (i.e., hydrogen), however, quantum effects become quite significant. To make the TST still valid for such low temperature reactions, many approaches have been proposed to quantize it [4-14]. However, there is no absolutely unambiguous way to do it.
To develop a more accurate and less ad hoc quantum version of TST, with a specific focus on the tunneling regime, Miller et al. [15-17] have proposed a quantum instanton (QI) approach recently. The QI is based on an earlier semiclassical (SC) TST [18] that became known as the instanton $[19,20]$. The similarity between the QI and SC instanton lies in using the steepest descent approximation to evaluate relevant integrals in the quantum rate formula, while the crucial difference is that the Boltzmann operator is evaluated by the quantum mechanics and semiclassical approximation, respectively. The QI theory thus incorporates the tunneling, corner cutting [21-24], and anharmonicity correctly and is expected to overcome the quantitative deficiency of the SC instanton model. In particular, the QI theory considers all tunneling paths and automatically gives each path its naturally weight factor from the quantum Boltzmann operator, instead of choosing a single optimal tunneling path, which is taken into account in the SC instanton and TSTs with SC tunneling corrections. Indeed, it has been numerically demonstrated that the QI predicts accurate quantum rates for one-dimensional and 
two-dimensional models within $20 \%$ error over a wide temperature range, from the deep tunneling to overbarrier regimes.

A lot of developments and applications [25-39] have been made since the QI theory is proposed. The original QI [15] involves the second time derivative of the flux-flux correlation at time zero. It has been further improved [29] by taking into account the higher derivatives of the flux-flux function. For the $1 \mathrm{D}$ and collinear reactions, the improved model is considerable accuracy, giving the rates to within $5 \%-10 \%$ errors. For a practical purpose, a simple and general way for choosing dividing surfaces used in the QI is suggested [31], namely, using the family of (hyper)planes normal to the minimum energy path at various distances s. A "simplest" QI model [26] has also been suggested with one dividing surface, slightly less accurate than the original QI. To reveal the relationship with conventional TSTs, the classical limit of the QI has been derived [37]. It is found that the classical TST is just a special case of the QI in high temperature limit; moreover, the quantum correction of the prefactor is more important than that of the activation energy in the TST.

Since the QI solely involves the Boltzmann operator and its relevant quantities, it can be applied to quite complex molecular systems (from gas phase [17, 37], liquid [28], to surface $[38,39]$ ) via well-established imaginary time path integral techniques. The first implementation of QI with path integral Monte Carlo and adaptive umbrella sampling techniques is applied to the three-dimensional hydrogen exchange reaction $\mathrm{D}+\mathrm{H}_{2} \rightarrow \mathrm{HD}+\mathrm{H}$ [16]. Soon, the techniques are further extended to the reaction of $\mathrm{H}+\mathrm{CH}_{4} \rightarrow$ $\mathrm{H}_{2}+\mathrm{CH}_{3}$ [17]. The thermodynamic integration with respect to the mass of the isotope and the inverse temperature is also proposed to compute the kinetic isotope effects [30] and rate constants [34] directly. To improve the convergence of the Monte Carlo simulation, the efficient "virial" estimators [32] have been derived from the logarithmic derivatives of the partition function and the delta-delta correlation functions, and it is found that their statistical errors do not increase with the number of discrete time slices in the path integral. Most recently, the QI has been compared with other conventional approaches [36] for an intramolecular proton transfer on a full-dimensional potential energy surface that incorporates high-levels $a b$ initio calculations along the reaction path. The obtained kinetic isotope effects from the QI are in reasonable agreement with those from the path-integral quantum TST.

In this paper, we firstly illustrate the QI formula and its path integral representation. Then, we display several applications, which are mainly done by ourselves. The systems include two gas phase reactions $\mathrm{H}+\mathrm{CH}_{4} \rightarrow \mathrm{H}_{2}+$ $\mathrm{CH}_{3}$ [17] and $\mathrm{H}+\mathrm{SiH}_{4} \rightarrow \mathrm{H}_{2}+\mathrm{SiH}_{3}$ [37], $\mathrm{H}$ diffusion on $\mathrm{Ni}(100)$ surface [38], and surface-subsurface transport and interior migration for $\mathrm{H} / \mathrm{Ni}[39]$.

\section{Method}

In this section, we summarize the rate formula for the QI evaluation. The detailed derivation can be found in [15-17]. The QI model proposes the following thermal rate constant:

$$
k_{\mathrm{QI}}=\frac{1}{Q_{r}} \frac{\sqrt{\pi} \hbar}{2} \frac{C_{f f}(0)}{\Delta H(\beta)} .
$$

Here, $Q_{r}$ is the reactant partition function per unit volume. $C_{f f}(0)$ is zero time value of the flux-flux correlation function

$$
C_{f f}(t)=\operatorname{tr}\left[e^{-\beta \hat{H} / 2} \hat{F}_{a} e^{-\beta \hat{H} / 2} e^{i \hat{H} t / \hbar} \hat{F}_{b} e^{-i \hat{H} t / \hbar}\right],
$$

where $\beta$ is the inverse temperature $1 /\left(k_{B} T\right), \hat{H}$ is the Hamiltonian operator of the reaction system, and $\hat{F}_{a}$ and $\hat{F}_{b}$ are the flux operators given by

$$
\widehat{F}_{\gamma}=\frac{i}{\hbar}\left[\hat{H}, h\left(s_{\gamma}(\hat{r})\right)\right]
$$

with $\gamma=a, b$. In (3), $h$ is the step-side function, $r$ represents the Cartesian coordinates of the reaction system, and $s_{a}(r)$ and $s_{b}(r)$ define two separate dividing surfaces via the equations $s_{a}(r)=0$ and $s_{b}(r)=0$, both $s_{a}(r)$ and $s_{b}(r)$ being positive (negative) on the product (reactant) side of the dividing surfaces.

$\Delta H(\beta)$ in (1) is a specific type of energy variance given by

$$
\Delta H(\beta)=\hbar \sqrt{\frac{-\ddot{C}_{d d}(0)}{2 C_{d d}(0)}} .
$$

In order to get the correct free particle (high temperature) limit (that would be $25 \%$ too large otherwise), an ad hoc term is added to $\Delta H(\beta), \Delta H_{\bmod }(\beta)=\Delta H(\beta)+(\sqrt{\pi}-$ $\sqrt{2}) / \beta$, which has very little effect in the low temperature regime. $C_{d d}(0)$ and $\ddot{C}_{d d}(0)$ are zero time value and its second time derivative, respectively, of the "delta-delta" correlation function

$$
C_{d d}(t)=\operatorname{tr}\left[e^{-\beta \hat{H} / 2} \Delta\left(s_{a}(\hat{r})\right) e^{-\beta \hat{H} / 2} e^{i \hat{H} t / \hbar} \Delta\left(s_{b}(\hat{r})\right) e^{-i \hat{H} t / \hbar}\right],
$$

where the generalized delta-function operator is

$$
\Delta\left(s_{\gamma}(\hat{r})\right)=\delta\left(s_{\gamma}(\hat{r})\right) \sqrt{\sum_{i=1}^{N} \frac{1}{m_{i}}\left(\nabla_{i} s_{\gamma}(\hat{r})\right)^{2}} \quad(\gamma=a, b) .
$$

Here, $N$ is the total number of atoms, $\nabla_{i}=\partial / \partial r_{i}, r_{i}$ denotes the Cartesian coordinates of the $i$ th atom, and $m_{i}$ is its atomic mass.

The dividing surfaces are determined by the stationary condition

$$
\frac{\partial}{\partial c_{k}} C_{d d}\left(0 ;\left\{c_{k}\right\}\right)=0
$$

where $\left\{c_{k}\right\}$ is a collection of parameters that is involved in the location of the dividing surfaces. This condition originates from the SC instanton model, and the resulting dividing surfaces correspond qualitatively to the turning points of the periodic orbit that runs on an upside down PES in imaginary time (see Appendix A in $[15,18]$ ). 
Since all the relevant quantities in the QI expression (1) involved only the quantum Boltzmann operator, they can be readily evaluated using imaginary time path integral Monte Carlo (PIMC) [40] method.

We begin with the simplest quantity, $C_{d d}(0)$, which can be discretized according to the standard procedure [41-43] to give

$$
\begin{aligned}
C_{d d}(0)= & C \int d r^{(1)} \int d r^{(2)} \cdots \int d r^{(P)} \Delta\left(s_{a}\left(r^{(0)}\right)\right) \\
& \times \Delta\left(s_{b}\left(r^{(P / 2)}\right)\right) \exp \left[-\beta \Phi\left(\left\{r^{(s)}\right\}\right)\right],
\end{aligned}
$$

where $C$ is a multiplicative constant, $P$ the number of imaginary time slices, and $r^{(s)}=\left(r_{1}^{(s)}, r_{2}^{(s)}, \ldots, r_{N}^{(s)}\right)$ the Cartesian coordinates of the system associated with the $s$ th time slice. $\Phi\left(\left\{r^{(s)}\right\}\right)$ is the discretized action given by

$$
\Phi\left(\left\{r^{(s)}\right\}\right)=\frac{P}{2 \hbar^{2} \beta^{2}} \sum_{s=1}^{P} \sum_{i=1}^{N} m_{i}\left(r_{i}^{(s)}-r_{i}^{(s-1)}\right)^{2}+\frac{1}{P} \sum_{s=1}^{P} V\left(r^{(s)}\right),
$$

where $r^{(0)}=r^{(P)}$ and $\left\{r^{(s)}\right\}$ represents $\left\{r^{(1)}, r^{(2)}, \ldots, r^{(P)}\right\}$. Path integral expressions for $C_{f f}(0)$ and $\ddot{C}_{d d}(0)$ are somewhat more complicated but can be obtained in a straightforward manner. The appropriate expressions are

$$
\begin{aligned}
C_{f f}(0)= & C \int d r^{(1)} \int d r^{(2)} \cdots \int d r^{(P)} \Delta\left(s_{a}\left(r^{(0)}\right)\right) \\
& \times \Delta\left(s_{b}\left(r^{(P / 2)}\right)\right) \exp \left[-\beta \Phi\left(\left\{r^{(s)}\right\}\right)\right] f_{v}\left(\left\{r^{(s)}\right\}\right),
\end{aligned}
$$

with

$$
\begin{aligned}
f_{v}\left(\left\{r^{(s)}\right\}\right)= & \left(\frac{i P}{2 \hbar \beta}\right)^{2} \frac{\sum_{i=1}^{N} \nabla_{i} s_{a}\left(r^{(0)}\right) \cdot\left(r_{i}^{(1)}-r_{i}^{(P-1)}\right)}{\sqrt{\sum_{i=1}^{N} m_{i}^{-1}\left(\nabla_{i} s_{a}\left(r^{(0)}\right)\right)^{2}}} \\
& \times \frac{\sum_{i=1}^{N} \nabla_{i} s_{b}\left(r^{(P / 2)}\right) \cdot\left(r_{i}^{(P / 2+1)}-r_{i}^{(P / 2-1)}\right)}{\sqrt{\sum_{i=1}^{N} m_{i}^{-1}\left(\nabla_{i} s_{b}\left(r^{(P / 2)}\right)\right)^{2}}},
\end{aligned}
$$

$$
\begin{aligned}
\ddot{C}_{d d}(0)= & -\frac{1}{\hbar^{2}} C \int d r^{(1)} \int d r^{(2)} \cdots \int d r^{(P)} \Delta\left(s_{a}\left(r^{(0)}\right)\right) \\
& \times \Delta\left(s_{b}\left(r^{(P / 2)}\right)\right) \exp \left[-\beta \Phi\left(\left\{r^{(s)}\right\}\right)\right] \\
& \times\left\{F\left(\left\{r^{(s)}\right\}\right)^{2}+G\left(\left\{r^{(s)}\right\}\right)\right\},
\end{aligned}
$$

with

$$
\begin{aligned}
F\left(\left\{r^{(s)}\right\}\right)= & -\frac{P}{\hbar^{2} \beta^{2}}\left\{\sum_{s=1}^{P / 2}-\sum_{s=P / 2+1}^{P}\right\} \sum_{i=1}^{N} m_{i}\left(r_{i}^{(s)}-r_{i}^{(s-1)}\right)^{2} \\
& +\frac{2}{P}\left\{\sum_{s=1}^{P / 2-1}-\sum_{s=P / 2+1}^{P-1}\right\} V\left(r^{(s)}\right), \\
G\left(\left\{r^{(s)}\right\}\right)= & \frac{2 f P}{\beta^{2}}-\frac{4 P}{\hbar^{2} \beta^{3}} \sum_{s=1}^{P} \sum_{i=1}^{N} m_{i}\left(r_{i}^{(s)}-r_{i}^{(s-1)}\right)^{2},
\end{aligned}
$$

with $f$ being the total number of degrees of freedom (i.e., $f=3 N)$.

In realistic calculations, we rewrite (1) as the product of several ratios

$$
k_{\mathrm{QI}}=\frac{\sqrt{\pi} \hbar}{2} \frac{C_{d d}(0)}{Q_{r}} \frac{C_{f f}(0)}{C_{d d}(0)} \frac{1}{\Delta H(\beta)} .
$$

The terms of $C_{f f}(0) / C_{d d}(0)$ and $\Delta H$ are directly calculated as a constrained average over the same ensemble of paths $[16,17]$

$$
\begin{gathered}
\frac{C_{f f}(0)}{C_{d d}(0)}=\left\langle f_{v}\right\rangle, \\
\Delta H^{2}=\frac{1}{2}\left\langle F^{2}+G\right\rangle,
\end{gathered}
$$

with

$$
\langle\cdots\rangle=\frac{\int d r^{(1)} \int d r^{(2)} \cdots \int d r^{(P)} \Delta\left(s_{a}\left(r^{(0)}\right)\right) \Delta\left(s_{b}\left(r^{(P / 2)}\right)\right) \exp \left[-\beta \Phi\left(\left\{r^{(s)}\right\}\right)\right](\cdots)}{\int d r^{(1)} \int d r^{(2)} \cdots \int d r^{(P)} \Delta\left(s_{a}\left(r^{(0)}\right)\right) \Delta\left(s_{b}\left(r^{(P / 2)}\right)\right) \exp \left[-\beta \Phi\left(\left\{r^{(s)}\right\}\right)\right]} .
$$

The evaluation of $C_{d d}(0) / Q_{r}$, however, meets a challenge because the $C_{d d}(0)$ is the quantity associated with the transition state, while $Q_{r}$ with the asymptotic reactant domain, we evaluate it using adaptive umbrella sampling techniques [44].

The QI approximation uses the short-time information of the flux-flux correlation function. Predescu and Miller [45] demonstrate that in the classical limit, Wigner's variational principle and the quantum variational criterion based on the minimization of flux-flux correlation function produce the same optimal surface. Recently, Wang et al. [37] have shown that in the classical limit, the QI formula is the same as the classical transition state theory. These situations motivate us to write the QI formula (13) as the Arrhenius form

$$
k_{\mathrm{QI}}=A_{\mathrm{QI}} \cdot e^{-\beta \Delta F} .
$$


Here, the free energy $\Delta F$ is defined by

$$
\Delta F=\frac{-1}{\beta} \cdot \log \left(\frac{C_{d d}(0)}{Q_{r}}\right) .
$$

This two-dimensional free energy is related to $Q_{r}$ (the reactant partition function) and $C_{d d}\left(0 ; \xi_{a}, \xi_{b}\right)$, and it is corresponding to the quality of probability density at $\left(\xi_{a}, \xi_{b}\right) . C_{d d}\left(0 ; \xi_{a}, \xi_{b}\right)$ has a similar property to the partition function at the transition state. The prefactor is given by

$$
A_{\mathrm{QI}}=\frac{\sqrt{\pi}}{2} \frac{C_{f f}(0)}{C_{d d}(0)} \frac{\hbar}{\Delta H} .
$$

The advantage of (16) allows us to investigate the respective quantum contributions to the rates from the quantum free energy and prefactor by comparing their quantum and classical values, since other factors such as the vibrationalrotational coupling and anharmonicity are automatically involved.

\section{Results and Discussions}

3.1. The Reaction of $\mathrm{H}+\mathrm{CH}_{4} \rightarrow \mathrm{H}_{2}+\mathrm{CH}_{3}$. The $\mathrm{H}+\mathrm{CH}_{4}$ reaction is a prototype of polyatomic hydrogen abstraction reaction. Quantum dynamical studies of this reaction have become possible only recently, because it involves 12 internal degrees of freedom and thus poses difficulties to quantum dynamics calculations as well as construction of the potential surface. We apply the QI methodology to this reaction using the potential energy surface constructed by Espinosa-García [46]. All calculations are performed in terms of the Cartesian coordinates of all the atoms (i.e., 18 degrees of freedom).

In the path integral simulations, the number of imaginary time slices $P$ is chosen to be 20 and 100 at temperatures $T=1000 \mathrm{~K}$ and $200 \mathrm{~K}$, respectively, while $3 \times 10^{7}$ Monte Carlo cycles are run to achieve $<10 \%$ statistical convergence.

3.1.1. Reaction Coordinate. For this reaction, we define a generalized reaction coordinate $s(r ; \xi)$, where $\xi$ is an adjustable parameter that shifts the location of the dividing surface (defined by $s(r ; \xi)=0) . s(r ; \xi)$ is defined by a linear interpolation between two constituent reaction coordinates $s_{0}(r)$ and $s_{1}(r)$ through the parameter $\xi$,

$$
s(r ; \xi)=\xi \cdot s_{1}(r)+(1-\xi) \cdot s_{0}(r)
$$

$s_{1}(r)$ is a reaction coordinate whose dividing surface is designed to pass through the top of the classical potential barrier, which is defined here as

$$
s_{1}(r)=\max \left\{s_{\alpha}(r), s_{\beta}(r), s_{\gamma}(r), s_{\delta}(r)\right\},
$$

with $s_{x}(r)(x=\alpha, \beta, \gamma, \delta)$ being the reaction coordinate that describes the abstraction process of one of the methane hydrogens $H_{x}$ by the incident one $H$

$$
s_{x}(r)=r\left(\mathrm{C}-\mathrm{H}_{x}\right)-r\left(\mathrm{H}_{x}-\mathrm{H}\right)-\left[r^{\dagger}\left(\mathrm{C}-\mathrm{H}_{x}\right)-r^{\dagger}\left(\mathrm{H}_{x}-\mathrm{H}\right)\right] \text {, }
$$

where $r(X-Y)$ denotes the interatomic distance between atoms $X$ and $Y$ and $r^{\dagger}(X-Y)$ is the value at the transition state geometry. $s_{0}(r)$, on the other hand, describes a dividing surface that is located far in the asymptotic reactant valley, which is given by

$$
s_{0}(r)=R_{\infty}-|R|
$$

Here, $R$ is the scattering vector that connects the incident hydrogen and the center of mass of the methane. $R_{\infty}$ is an adjustable parameter which is chosen to be $9 \AA$ in order to guarantee that the interaction potential energy between $\mathrm{H}$ and $\mathrm{CH}_{4}$ is negligible.

Now, the term $C_{d d}(0)$ (5) becomes a function of two parameters, $\xi_{a}$ and $\xi_{b}$, as follows:

$$
C_{d d}\left(0 ; \xi_{a}, \xi_{b}\right)=\operatorname{tr}\left[e^{-\beta \hat{H} / 2} \Delta\left(s\left(\hat{r} ; \xi_{a}\right)\right) \times e^{-\beta \hat{H} / 2} \Delta\left(s\left(\hat{r} ; \xi_{b}\right)\right)\right],
$$

and thus, one seeks a stationary point of $C_{d d}\left(0 ; \xi_{a}, \xi_{b}\right)$ in the two-dimensional $\left(\xi_{a}, \xi_{b}\right)$ space to obtain the corresponding “optimal" values.

3.1.2. Free Energy Surface. The quantity $C_{d d}\left(0 ; \xi_{a}, \xi_{b}\right)$ varies exponentially as a function of $\left(\xi_{a}, \xi_{b}\right)$, it is convenient to define a quantum "free energy surface" as follows:

$$
F\left(\xi_{a}, \xi_{b}\right)=-k_{B} T \log C_{d d}\left(0 ; \xi_{a}, \xi_{b}\right),
$$

and locate the saddle point of $F\left(\xi_{a}, \xi_{b}\right)$ by visual inspection.

Figure 1 shows the free energy surfaces for the $\mathrm{H}+\mathrm{CH}_{4}$ reaction. It exhibits a barrier-like profile along the direction $\bar{\xi}=\left(\xi_{a}+\xi_{b}\right) / 2$, while it grows approximately quadratically with the increasing of the absolute value of $\Delta \xi=\xi_{a}-\xi_{b}$. From this figure, it is seen that at a higher temperature $T=$ $1000 \mathrm{~K}$ (Figure $1(\mathrm{a})$ ), there appears only a single saddle point at $(\bar{\xi}, \Delta \xi)=(1.02,0.0)$, while at a low temperature $T=200 \mathrm{~K}$ (Figure 1(b)), the saddle point bifurcates into a distinct pair at $(\bar{\xi}, \Delta \xi)=(1.1, \pm 0.35)$, which indicates the existence of nonnegligible tunneling effects in the rate constant $[15,16]$.

3.1.3. Rate Constants. Having obtained the "optimal" values of the $\left(\xi_{a}, \xi_{b}\right)$ at each temperature, one can now compute the quantum instanton rate by combining various quantities as in (8). The calculated QI rates are tabulated in Table 1 as well as other theoretical and experimental ones.

Comparing the quantum instanton rates with others, we find that $k_{\mathrm{QI}}$ is in good agreement with the experimental data more specifically, it is closer to the rates obtained by Baulch et al. [47] than those by Sutherland et al. [48], and $k_{\mathrm{QI}}$ agrees with $k_{\mathrm{CVT} / \mu \mathrm{OMT}}$ within $10 \%$ for the temperature range $T=$ $600-2000 \mathrm{~K}$, but it becomes somewhat larger than the latter as the temperature is decreased (the deviation becomes $30 \%$ and $55 \%$ for $T=500$ and $300 \mathrm{~K}$, resp.). It should be noted that the differences between $k_{Q I}$ and $k_{\mathrm{CVT} / \mu \mathrm{OMT}}$ are much smaller than the uncertainty of the experimental data.

Figure 2 displays the Arrhenius plots of the rate constants. In Figure 2, we also plot the accurate quantum dynamics results of $\mathrm{MCTDH}$ (the multiconfigurational 


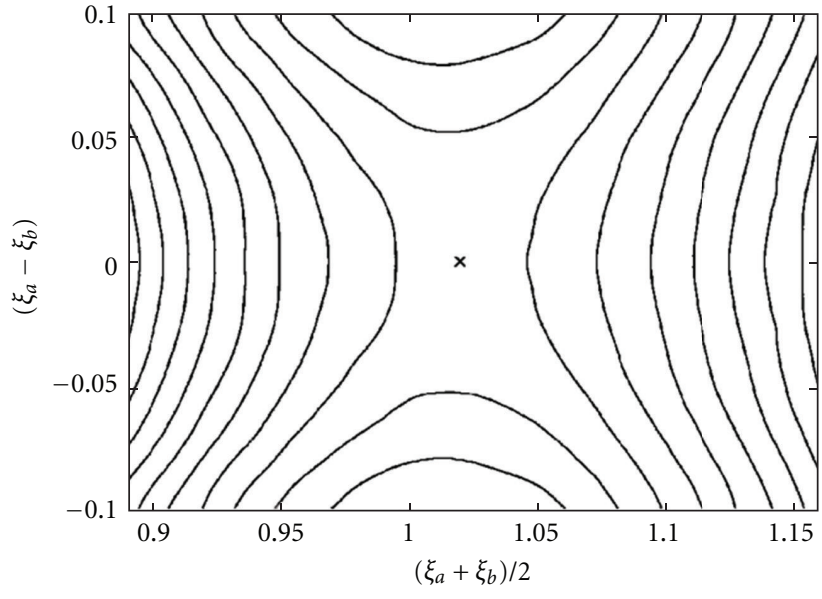

(a)

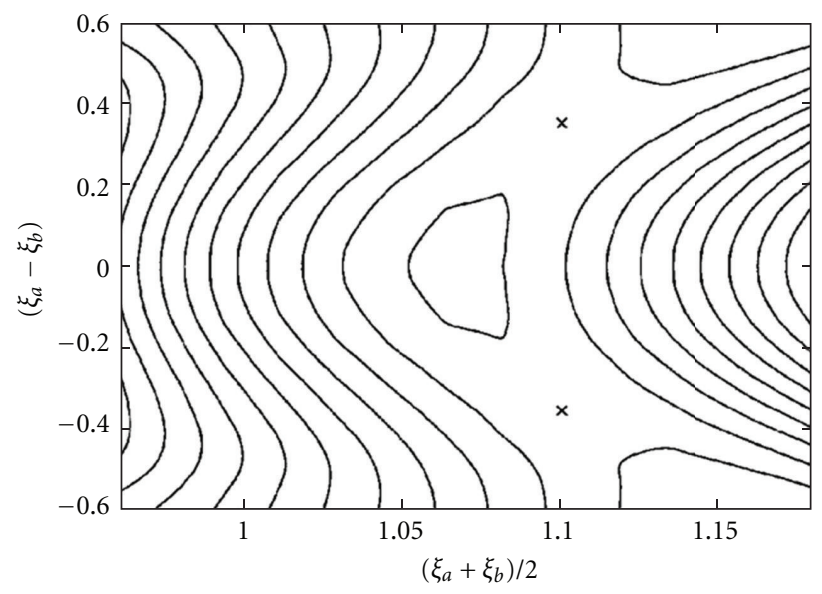

(b)

Figure 1: Local topography of the quantum free energy surface defined by (24) near the top of the barrier. (a) $T=1000 \mathrm{~K}$; (b) $T=200 \mathrm{~K}$. The cross symbols show the location of the saddle points. The values of $\xi_{a}$ and $\xi_{b}$ at the saddle points are used as input for computing the quantum instanton rate.

TABLE 1: Theoretical and experimental rate constants obtained for the $\mathrm{H}+\mathrm{CH}_{4} \rightarrow \mathrm{H}_{2}+\mathrm{CH}_{3}$ reaction. $\left(\xi_{a}\right.$, $\left.\xi_{b}\right)$ is the saddle point of the quantum free energy surface. $k_{\mathrm{QI}}$ is the quantum instanton rate constant. $k_{\mathrm{CVT} / \mu \mathrm{OMT}}$ is the rate constant of the canonical variational theory with microcanonical optimized multidimensional tunneling (CVT/ $\mu \mathrm{OMT})$ [46]. $k_{\exp t}^{a}$ and $k_{\exp t}^{b}$ are the experimental Arrhenius fits, $k(T)=2.18 \times 10^{-20} T^{3.0} \exp (-4045 / T)[47]$ and $k(T)=6.78 \times 10^{-21} T^{3.156} \exp (-4406 / T)$ [48], respectively. Unit: $\mathrm{cm}^{3} \mathrm{~s}^{-1}$ for rates.

\begin{tabular}{|c|c|c|c|c|c|}
\hline$T(\mathrm{~K})$ & $\left(\xi_{a}, \xi_{b}\right)$ & $k_{\mathrm{QI}}$ & $k_{\mathrm{CVT} / \mu \mathrm{OMT}}$ & $k_{\exp t}^{a}$ & $k_{\exp t}^{b}$ \\
\hline 200 & $(0.93,1.28)$ & $3.30(-22)$ & & $2.87(-22)$ & $3.36(-23)$ \\
\hline 300 & $(1.05,1.05)$ & $7.80(-19)$ & $5.03(-19)$ & $8.20(-19)$ & $1.86(-19)$ \\
\hline 400 & $(1.05,1.05)$ & $6.00(-17)$ & $4.16(-17)$ & $5.66(-17)$ & $1.82(-17)$ \\
\hline 500 & $(1.04,1.04)$ & $9.00(-16)$ & $6.96(-16)$ & $8.35(-16)$ & $3.33(-16)$ \\
\hline 600 & $(1.03,1.03)$ & $5.42(-15)$ & $5.00(-15)$ & $5.56(-15)$ & $2.57(-15)$ \\
\hline 700 & $(1.02,1.02)$ & $2.40(-14)$ & & $2.31(-14)$ & $1.19(-14)$ \\
\hline 800 & $(1.02,1.02)$ & $7.13(-14)$ & $6.72(-14)$ & $7.10(-14)$ & $4.00(-14)$ \\
\hline 900 & $(1.02,1.02)$ & $1.85(-13)$ & & $1.78(-13)$ & $1.07(-13)$ \\
\hline 1000 & $(1.02,1.02)$ & $3.97(-13)$ & $3.58(-13)$ & $3.82(-13)$ & $2.43(-13)$ \\
\hline 2000 & $(1.00,1.00)$ & $1.52(-11)$ & $1.70(-11)$ & $2.31(-11)$ & $1.96(-11)$ \\
\hline
\end{tabular}

time-dependent Hartree approach) [49]. Compared to the MCTDH ones, our QI rate constants are larger by factors of about 2 to 3 over the temperature range $300-400 \mathrm{~K}$. This difference may partly be due to the recrossing effect which is not considered in QI theory and partly arise from the use of the J-shifting approximation and the neglect of the vibrational angular momenta Hamiltonian in MCTDH method [49].

3.2. The Reaction of $\mathrm{H}+\mathrm{SiH}_{4} \rightarrow \mathrm{H}_{2}+\mathrm{SiH}_{3}$. The $\mathrm{H}+\mathrm{SiH}_{4}$ reaction is an important step in the radical mechanism of thermal decomposition of monosilane. We calculate the rates and kinetic isotope effects (KIEs) of this reaction with the quantum instanton method in full Cartesian space, on the basis of analytical potential energy surface constructed by Espinosa-García et al. [50].

The reaction coordinate of $\mathrm{H}+\mathrm{SiH}_{4}$ has the same form as that of $\mathrm{H}+\mathrm{CH}_{4}$ (Section 3.1.1). In our QI calculations, the number of time slices, $P$, is set to be $20(1000 \mathrm{~K})$ and $120(200 \mathrm{~K})$ for the quantum evaluations. In our classical evaluations, the formula is the same as QI, but the number of time slices in the path integral is set to be 1 . The number of Monte Carlo is about $(6-10) \times 10^{6}$ for computing a single ensemble average, and it converges the relevant quantities within $10 \%$ statistical errors.

3.2.1. Free Energy and Prefactor. We have rewritten the QI formula (8) in the Arrhenius form (16), which consists of the free energy and prefactor. In this section, we calculate the corresponding quantum and classical quantities so as to investigate the respective quantum contributions to the rates from the quantum free energy and prefactor. Our calculated results are plotted in Figures 3 and 4.

Now, we look into the quantum effects from both the free energy and prefactor. Figure 3 plots the quantum and classical free energies as a function of temperature 


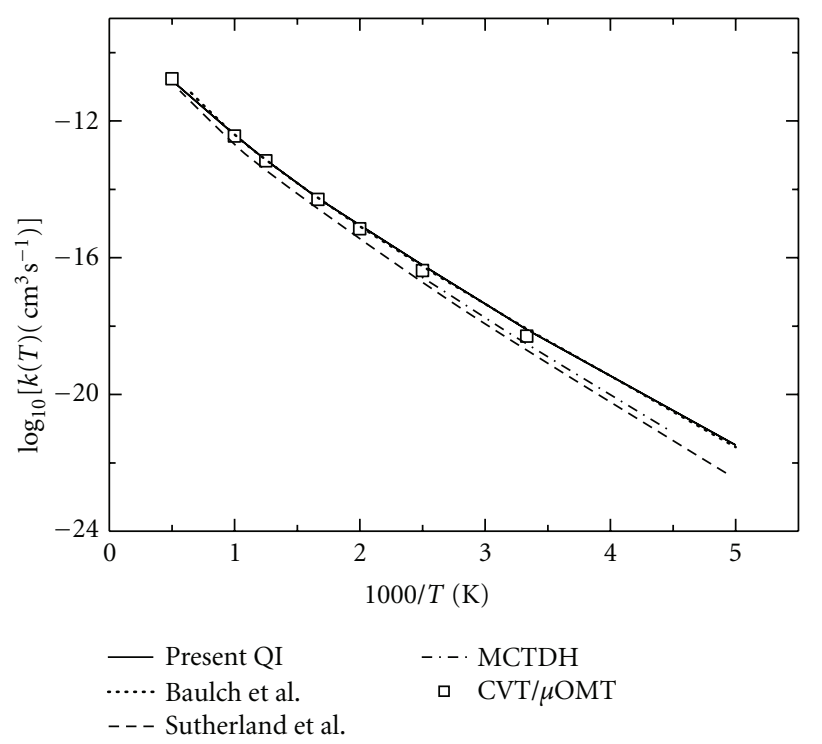

Figure 2: Arrhenius plots of the thermal rate constant for the $\mathrm{H}+\mathrm{CH}_{4} \rightarrow \mathrm{H}_{2}+\mathrm{CH}_{3}$ reaction. The solid line is the quantum instanton rate. Dotted and dashed lines are the Arrhenius fits of the experimental data from $[47,48]$, respectively. The dot-dashed line is the result of the multiconfigurational time-dependent Hartree approach (MCTDH) [49]. The open squares are the results of the canonical variational theory with microcanonical optimized multidimensional tunneling (CVT/ $\mu \mathrm{OMT})[46]$.

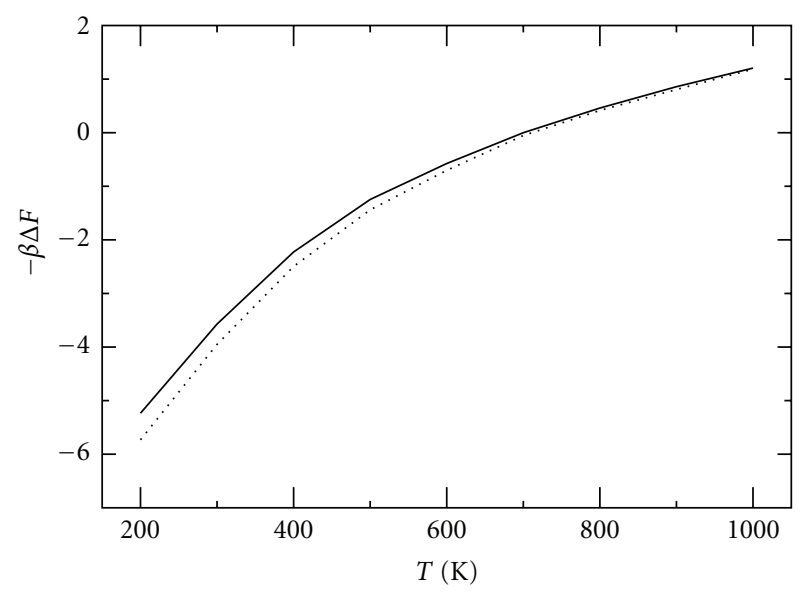

Figure 3: The temperature dependence of the free energy. The solid and dotted lines correspond to the quantum and classical calculations, respectively.

at the optimized stationary point. One immediately observes that the quantum effect becomes significant at $T<600 \mathrm{~K}$, whereas both quantum and classical results nearly coincide at $T>600 \mathrm{~K}$. As expected, the quantum effect always decreases the classical free energy. The difference of the contributions to the rates is about $40 \%$ at $200 \mathrm{~K}$.

Figure 4 shows the temperature dependence of the quantum and classical prefactors. Compared with Figure 3, one easily finds that this quantum contribution is much larger than that for the free energy correction. Even at $1000 \mathrm{~K}$,

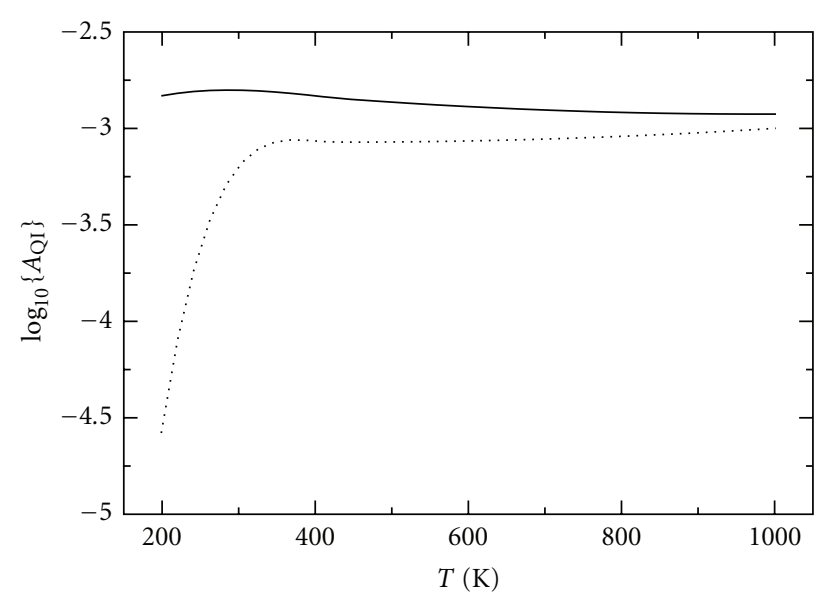

Figure 4: The temperature dependence of the prefactor. The solid and dotted lines correspond to the quantum and classical calculations, respectively.

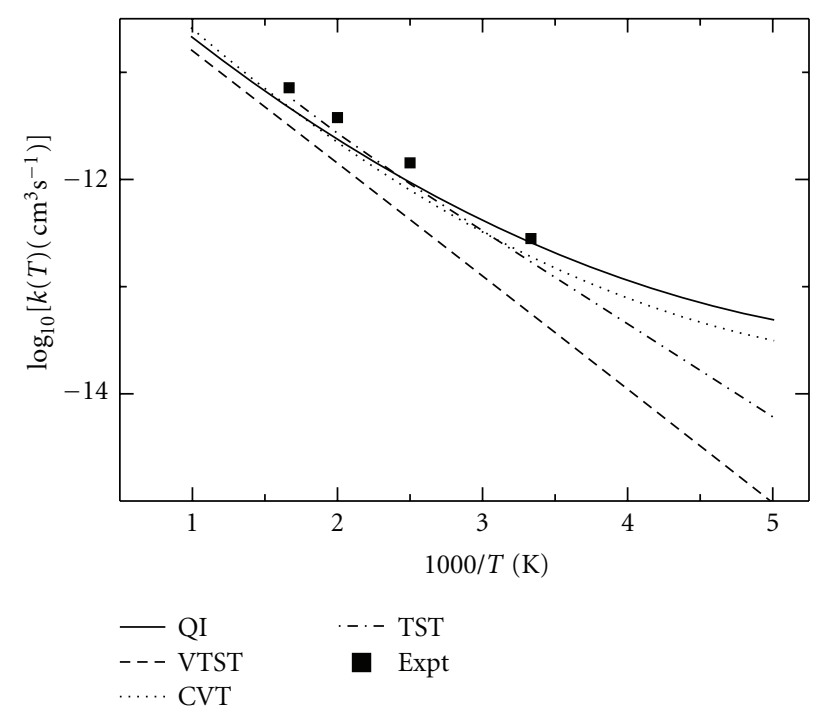

Figure 5: Arrhenius plot of the thermal rate constants for the $\mathrm{H}+\mathrm{SiH}_{4}$ reaction. Solid line, the QI results; dashed line, the classical VTST results; dotted line, CVT/CD-SCSAG results from [50]; dotdashed, the values from the conventional TST with a simple Wigner tunneling factor [51]; filled squares are the experimental values from [52].

the difference is observable. At $200 \mathrm{~K}$, the difference is about several orders of magnitude.

In the QI theory, it is not possible to explicitly distinguish the quantum contributions from the partition function and nuclear tunneling, but we can conclude that it is insufficient to estimate the accurate rate by only replacing the activation energy in the TST with its quantum analog, because the quantum prefactor plays more important rule in determining the quantum rate.

3.2.2. Rate Constants. In Table 2 , we summarize the rate constants obtained from the QI theory and classical VTST (this kind of results are performed by setting the time slices 
TABLE 2: Rate constants for the $\mathrm{H}+\mathrm{SiH}_{4}$ reaction $\left(\mathrm{cm}^{3} \mathrm{~s}^{-1}\right)$. The CVT/CD-SCSAG results from [50]. DD is the conventional TST with simple Wigner tunneling factor [51]. Expt. is the experimental Arrhenius fits, $k(T)=1.78 \times 10^{-10} \exp (-3820 / R T)(T=290-660 \mathrm{~K})[52]$.

\begin{tabular}{|c|c|c|c|c|c|}
\hline$T(\mathrm{~K})$ & Present QI & VTST & CVT/CD-SCSAG & $\mathrm{DD}$ & Expt. \\
\hline 200 & $4.82(-14)$ & $8.88(-16)$ & $3.07(-14)$ & $6.1(-15)$ & \\
\hline 300 & $2.74(-13)$ & $1.38(-14)$ & $2.09(-13)$ & $1.7(-13)$ & $2.81(-13)$ \\
\hline 400 & $9.67(-13)$ & $4.32(-13)$ & $7.96(-13)$ & $9.1(-13)$ & $1.43(-12)$ \\
\hline 500 & $2.37(-12)$ & $1.23(-12)$ & $2.11(-12)$ & $2.7(-12)$ & $3.78(-12)$ \\
\hline 600 & $3.99(-12)$ & $2.55(-12)$ & $4.43(-12)$ & $5.9(-12)$ & $7.18(-12)$ \\
\hline 700 & $7.60(-12)$ & $5.18(-12)$ & $8.00(-12)$ & & \\
\hline 800 & $1.18(-11)$ & $8.63(-12)$ & & & \\
\hline 900 & $1.76(-11)$ & $1.29(-11)$ & & & \\
\hline 1000 & $2.39(-11)$ & $1.99(-11)$ & $2.80(-11)$ & & \\
\hline
\end{tabular}

in the path integral to be 1 in the QI calculations) as well as those from the canonical variational TST with the centrifugal-dominant small curvature SC adiabatic groundstate (CVT/CD-SCSAG) approach [50], the conventional TST with simple Wigner tunneling factor [51] and the experiment [52]. The corresponding Arrhenius plots are displayed in Figure 5.

Table 2 and Figure 5 display comparable results of the CVT/CD-SCSAG to the QI values, with 36\% maximal errors at $200 \mathrm{~K}$ and a slightly different Arrhenius slope. Both results are in good agreement with experimental data in the tested temperature range. This manifests that the PES used is reasonable accurate. However, the values from the classical VTST are always smaller than the QI results especially in the deep tunneling regions. The conventional TST results have similar tendency to those from the classical VTST. Although the classical VTST can be much improved by evaluating the partition functions quantum mechanically, we do not focus on this improvement. However, it is noted that the anharmonicity, rotational-vibrational coupling are involved in the classical simulation. It is thus expected that these large errors come from the pure quantum effects.

3.2.3. Kinetic Isotope Effects. Kinetic isotope effect (KIE) is the characteristic of chemical reactions which may reveal the quantum effect. We consider the following isotopic reactions:

$$
\begin{array}{ll}
\text { (R1) } \mathrm{H}+\mathrm{SiH}_{4} \longrightarrow \mathrm{SiH}_{3}+\mathrm{H}_{2} \\
\text { (R2) } \mathrm{D}+\mathrm{SiH}_{4} \longrightarrow \mathrm{SiH}_{3}+\mathrm{HD} \\
\text { (R3) } \mathrm{H}+\mathrm{SiD}_{4} \longrightarrow \mathrm{SiD}_{3}+\mathrm{HD} .
\end{array}
$$

The calculated values are tabulated in Table 3.

The KIEs of $k_{\mathrm{QI}}(\mathrm{R} 1) / k_{\mathrm{QI}}(\mathrm{R} 2)$ and $k_{\mathrm{QI}}(\mathrm{R} 1) / k_{\mathrm{QI}}(\mathrm{R} 3)$ in the temperature range of 200-1000 $\mathrm{K}$ are summarized in Table 3. Besides the QI values, this table also displays comparable results of the CVT/CD-SCSAG [50]. It is easily found that the $k(\mathrm{R} 1) / k(\mathrm{R} 2)$ KIEs predicted by the QI are smaller than 1 , in agreement with the CVT/CD-SCSAG values. The detailed comparison reveals that the CVT/CD-SCSAG predicts smaller values than those from QI theory. Again, the maximal error occurs at $200 \mathrm{~K}$ and is about $23 \%$. For $k(\mathrm{R} 1) / k(\mathrm{R} 3)$ KIEs, we find that although both approaches predict the values larger than 1 at 200 to $1000 \mathrm{~K}$, the QI values are smaller than those from CVT/CD-SCSAG. EspinosaGarcía and coworkers [50] have pointed out that the CVT/CD-SCSAG rates may have been overestimated because of the high vibrational and tunneling contributions. This manifests that the QI approach indeed correctly accounts for the quantum effects.

3.3. H Diffusion on Ni(100) Surface. Diffusion plays a fundamental role in surface process. It reveals characteristics about the underlying surface potential and is intimately involved in determining the kinetics of surface catalyzed chemical reactions. The hydrogen atom and its isotopes are ideal candidates to exhibit quantum tunneling behavior due to their small masses. We explore the evaluation of the quantum instanton approximation to the process of $\mathrm{H}$ diffusion on $\mathrm{Ni}(100)$ surface using the EAM4 potential energy surface constructed by Truong and Truhlar [53].

In the path integral calculations, the numbers of time slices, $P$ and $P_{\text {bath }}$, for the degrees of freedom of $\mathrm{H}$ and quantized $\mathrm{Ni}$ atoms, respectively, are set to $\left(P, P_{\text {bath }}\right)=$ $(24-40,6-8)$ in the temperature range of $40-300 \mathrm{~K}$. The number of Monte Carlo cycle employed is $(1-10) \times 10^{6}$, which converges most of the relevant quantities within $10 \%$ statistical error (some of the statistical errors are within $20 \%$ below $100 \mathrm{~K}$ ).

3.3.1. Model. Nickel crystallizes in a face-centered-cubic (fcc) lattice structure. The structural model used consists of $162 \mathrm{Ni}$ atoms over four layers: 40 atoms are in each of the first and third layers, and 41 atoms are in each of the second and fourth layers.

Figure 6 shows the platform and profile chart of $\mathrm{Ni}(100)$ lattice. In the simulations, $89 \mathrm{Ni}$ atoms (orange), in the four sides of the bulk metal and the bottom layer, are fixed. The 48 atoms (blue) closest to the fixed ones are considered to be movable and treated classically. The last 25 ones (red), surrounding the reactant and product sites and lying directly beneath the reactant and product sites, are treated quantum mechanically.

In our calculations, the lattice (see Figure 6) is rotated by 45 degrees counterclockwise, and we chose the $x$ coordinate of $\mathrm{H}$ atom (along the reaction path) to be reaction coordinate. 
TABLE 3: The kinetic isotope effects (KIEs) at different temperatures. CVT is the CVT/CD-SCSAG values from [50].

\begin{tabular}{lcccc}
\hline$T(\mathrm{~K})$ & QI KIE $(R 1 / R 2)$ & CVT KIE $(R 1 / R 2)$ & QI KIE $(R 1 / R 3)$ & CVT KIE $(R 1 / R 3)$ \\
\hline 200 & 0.56 & 0.69 & 30.10 & 33.60 \\
300 & 0.73 & 0.71 & 8.57 & 9.81 \\
400 & 0.77 & 0.76 & 3.11 & 5.07 \\
500 & 0.85 & 0.81 & 2.41 & 3.46 \\
600 & 0.90 & 0.84 & 2.15 & 2.72 \\
700 & 0.92 & 0.86 & 1.78 & 2.32 \\
800 & 0.94 & & 1.68 & 1.80 \\
900 & 0.94 & & 1.64 & \\
1000 & 0.95 & 0.90 & & \\
\hline
\end{tabular}

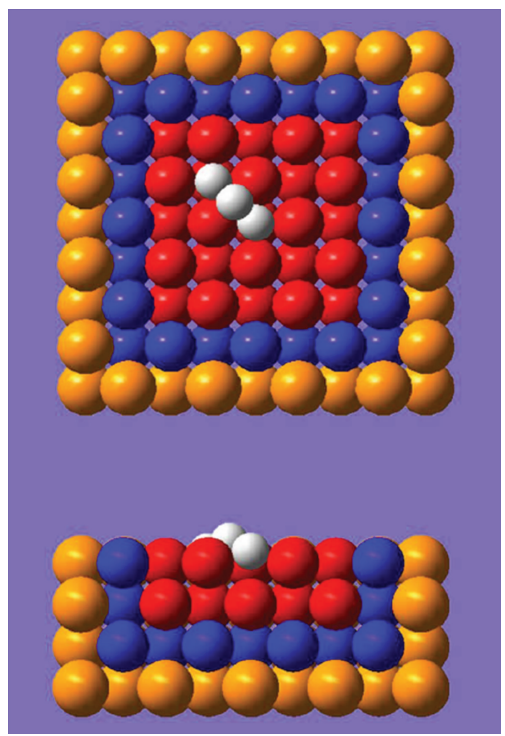

FIGURE 6: The platform and profile chart of $\mathrm{H}$ diffusion on $\mathrm{Ni}(100)$ lattice. The gray circles represent $\mathrm{H}$ atom on different surface sites, and the orange circles represent the fixed $\mathrm{Ni}$ atoms. Blue circles are $\mathrm{Ni}$ atoms treated classically, while the red ones are treated quantum mechanically.

3.3.2. Probability Distribution of Paths. The path integral has an advantage that the paths of the particles can display the character of the diffusive process. We extract the probability distributions for the paths of $\mathrm{H}$ and $\mathrm{Ni}$ atoms (two reaction coordinate beads $\left(x_{0}\right.$ and $\left.x_{P / 2}\right)$ of $\mathrm{H}$ path are fixed at the two dividing surfaces) for the purpose of guaranteeing the instanton property (the instanton is a periodic orbit between the two dividing surfaces). The dividing surfaces can be obtained from the free energy surface (24), their values at different temperatures are shown in Table 4.

Figure 7 displays the probability distributions for both $\mathrm{H}$ and $\mathrm{Ni}$ atoms at several temperatures. It is found that the probability distribution of $\mathrm{H}$ is localized at $300 \mathrm{~K}$, and it becomes more and more delocalized as the temperature decreases. Below $80 \mathrm{~K}$, the distribution rapidly becomes obvious in both sides of the transition state, and the path begins to continuously distribute between the two hollow sites at lower temperatures. This phenomenon indicates that

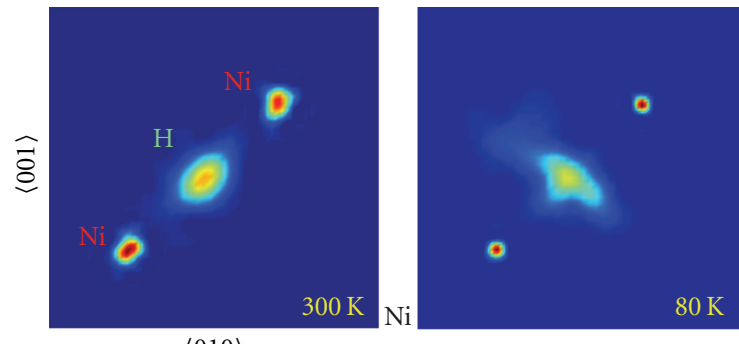

$\langle 010\rangle$
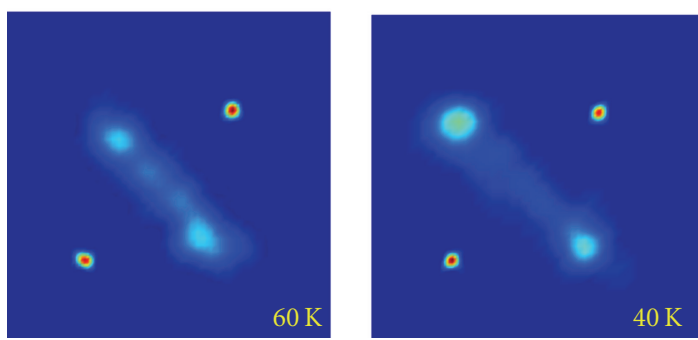

Figure 7: Distribution of $\mathrm{H}$ and Ni quantum paths on the surface with two reaction coordinate beads $\left(x_{0}\right.$ and $\left.x_{P / 2}\right)$ of $\mathrm{H}$ path fixed at the two dividing surfaces. Labels of $<010>$ and $<001>$ denote the crystal directions of nickel. The probabilities are normalized for both $\mathrm{H}$ and $\mathrm{Ni}$.

$\mathrm{H}$ tunneling becomes remarkable at the temperatures lower than $80 \mathrm{~K}$. The probability distributions of $\mathrm{Ni}$ atoms have small changes in the whole temperature range, and they seem to be frozen at very low temperature.

3.3.3. Diffusion Coefficients. According to the hopping model [54], the diffusion coefficient $D$ is related to the rates through

$$
D=k_{\mathrm{QI}} \cdot b^{2} \text {, }
$$

where $b$ is the hop length and is equal to $2.489 \AA$ for the $\mathrm{Ni}(100)$ surface. We have calculated the diffusion coefficients at temperatures ranging from 40 to $300 \mathrm{~K}$, and the results are plotted in Figure 8.

We plot two kinds of QI diffusion coefficients in Figure 8, one is the result on a rigid lattice, the other is the result on a quantized lattice. At high temperatures, these two kinds of diffusion coefficients are nearly the same; however, at low temperatures, the ones on a rigid lattice are bigger than that 
TABle 4: The positions of two dividing surfaces. (Unit in $\AA$. The position 0.00 corresponds to the bridge site and the positions $\pm 1.24 \AA$ correspond to two hollow sites.)

\begin{tabular}{lccccc}
\hline$T(\mathrm{~K})$ & 300 & 80 & 60 & 50 & 40 \\
\hline$x_{a}^{\dagger}$ & 0.00 & 0.00 & -0.75 & -1.00 & -1.08 \\
$x_{b}^{\dagger}$ & 0.00 & 0.00 & 0.75 & 1.00 & 1.08 \\
\hline
\end{tabular}

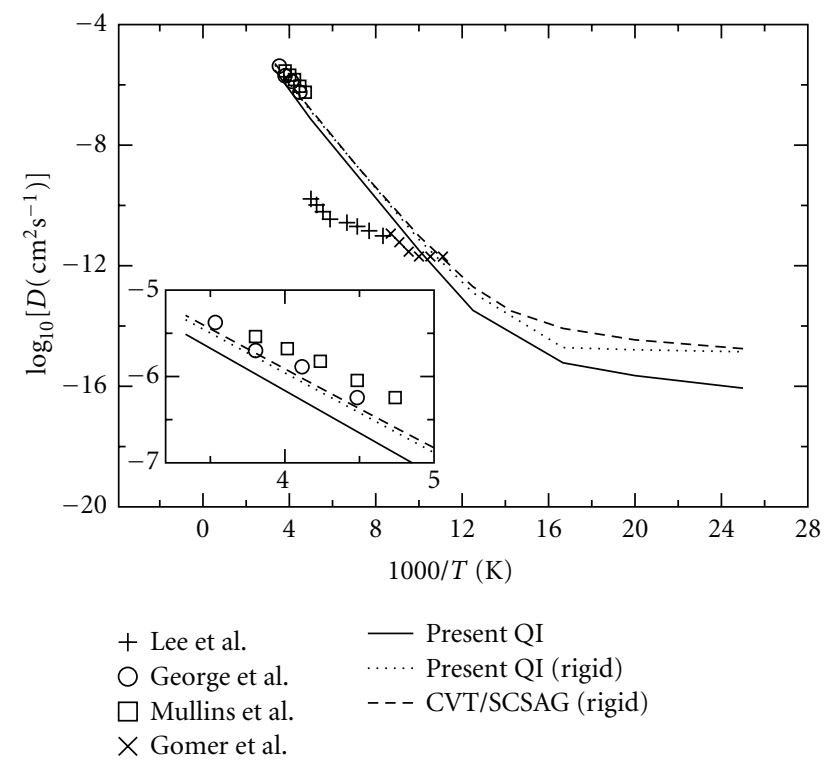

Figure 8: Arrhenius plots of the diffusion coefficients in the range 40-300 K. Solid line: the QI results for the quantized lattice; dotted line: the QI results for the rigid lattice; dashed line: the CVT/SCSAG results for the rigid lattice [55]. Pluses, circles, squares, and crosses are experimental results of Lee et al. [58], George et al. [56], Mullins et al. [57], and Lin and Gomer [59], respectively.

on a quantized lattice, and this is mainly due to the fact that the free energy on a quantized lattice is higher than that on a rigid lattice [38]. Comparing the QI diffusion coefficients with the CVT/SCSAG ones [55] on a rigid lattice, we find that our QI results on a rigid lattice are very similar to those from the CVT/SCSAG. Now, we compare the present theoretical results with experimental ones. It is found that the QI results as well as the CVT/SCSAG results are in good agreement with the experiments of George et al. [56] and Mullins et al. [57] at $200 \mathrm{~K}$ and $300 \mathrm{~K}$. However, at low temperatures, the theoretical results are significantly larger than Lee et al. [58] and Lin and Gomer [59]. The experimental transition temperature $(100 \mathrm{~K}-160 \mathrm{~K})$ is also different from the present calculations. The QI predicts it to be $70 \mathrm{~K}$ for the quantized lattice, while it is about $66 \mathrm{~K}$ for the rigid lattice obtained from both the QI and the CVT/SCSAG approaches. These discrepancies between theories and experiments may be attributed to the interaction potential. It is likely that the accuracy of the potential energy surfaces needs further improvement.

3.4. Surface-Subsurface Transport and Interior Migration for $\mathrm{H} / \mathrm{Ni}$. A full-dimensional potential energy surface (EAM6),

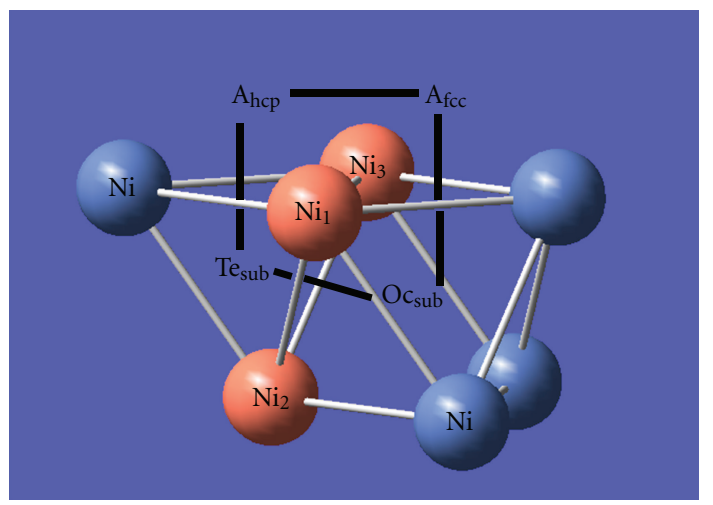

Figure 9: A lattice model with a few $\mathrm{Ni}$ atoms for the circle reaction processes of hydrogen. $\mathrm{A}_{\mathrm{hcp}}, \mathrm{A}_{\mathrm{fcc}}, \mathrm{Oc}_{\text {sub }}$, and $\mathrm{Te}_{\text {sub }}$ are the abbreviations for a hcp hollow site, a fcc hollow site, a subsurface octahedral vacancy, and a subsurface tetrahedral vacancy, respectively. The black lines stand for the directions of the reaction paths. The atoms of $\mathrm{Ni}_{1}, \mathrm{Ni}_{2}$, and $\mathrm{Ni}_{3}$ colored in orange are specially used to determine the reaction coordinate of the process $\mathrm{Oc}_{\mathrm{sub}} \rightarrow \mathrm{Te}_{\mathrm{sub}}$.

constructed by Wonchoba and Truhlar [60], using the embedded diatomics-in-molecules (EDIMs) [61] potential energy function, is used in this QI calculations.

In path integral calculations, the numbers of time slices, $P$ and $P_{\text {bath }}$ for the degrees of freedom of the $\mathrm{H}$ and quantized $\mathrm{Ni}$ atoms, respectively, are set to $\left(P, P_{\text {bath }}\right)=(30-40,6-8)$ in the temperature range of $100-400 \mathrm{~K}$. The number of Monte Carlo is about $(2-6) \times 10^{6}$ for computing a single ensemble average. It converges most of the values within $10 \%$ statistical errors (some of the statistical errors are within $20 \%$ at $100 \mathrm{~K}$ ).

3.4.1. Model. Figures 9 and 10 show $\mathrm{H}$ diffusion processes in the nickel crystal with a face-centered-cubic (fcc) lattice structure. For a given rate process, we construct a lattice cell (each cell contains more than $200 \mathrm{Ni}$ atoms) in which all atoms are treated to be movable to incorporate the effect of the crystal fluctuation on the rates. To be concrete, the $\mathrm{Ni}$ atoms in the four sides of the bulk metal and at the bottom layers are fixed, the $\mathrm{Ni}$ atoms surrounding the reactant and product sites and lying along the reaction path are treated quantum mechanically, and the others are treated classically. It should be noted that the lattice for the interior diffusion process has a structure of sphere and the outer layers are fixed.

In the QI calculations, we need to define the reaction coordinate operators $\hat{s}(6)$. For the systems considered in this section, the hydrogen coordinates are essentially good choices. We thus adopt the following reaction coordinates for different rate processes. In the $\mathrm{H}$ diffusion on $\mathrm{Ni}(111)$ surface 


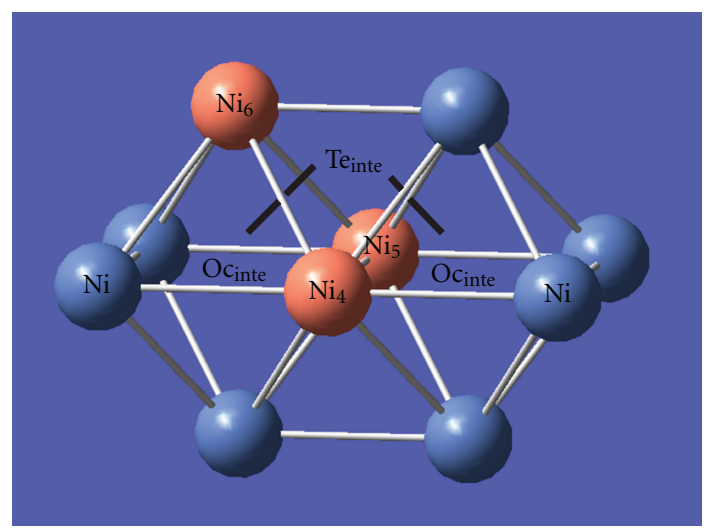

Figure 10: A lattice model with a few $\mathrm{Ni}$ atoms for the process of $\mathrm{H}$ diffusion in interior of bulk $\mathrm{Ni}$. $\mathrm{Oc}_{\text {inte }}$ and $\mathrm{Te}_{\text {inte }}$ are the abbreviations for an interior octahedral vacancy and an interior tetrahedral vacancy, respectively. The black lines stand for the general directions of the reaction paths. The atoms of $\mathrm{Ni}_{4}, \mathrm{Ni}_{5}$, and $\mathrm{Ni}_{6}$ colored in orange are specially used to determine the reaction coordinate of the process $\mathrm{Oc}_{\text {inte }} \rightarrow \mathrm{Te}_{\text {inte }}$.

from a hcp site to a fcc site $\left(\mathrm{A}_{\mathrm{hcp}} \rightarrow \mathrm{A}_{\mathrm{fcc}}\right)$, the $x$ coordinate of the $\mathrm{H}$ atom is chosen, whose direction is showed in Figure 9 as the black line connecting the $\mathrm{A}_{\mathrm{hcp}}$ site to $\mathrm{A}_{\mathrm{fcc}}$ site. In the $\mathrm{H}$ resurfacing from a subsurface octahedral vacancy to the $\mathrm{fcc}$ site $\left(\mathrm{Oc}_{\mathrm{sub}} \rightarrow \mathrm{A}_{\mathrm{fcc}}\right)$ and from a subsurface tetrahedral vacancy to the hcp site $\left(\mathrm{Te}_{\text {sub }} \rightarrow \mathrm{A}_{\mathrm{hcp}}\right)$, the $z$ coordinate of the $\mathrm{H}$ atom (vertical to $\mathrm{Ni}(111)$ surface) is taken. In the $\mathrm{H}$ diffusion between the adjacent subsurface octahedral and tetrahedral vacancies $\left(\mathrm{Oc}_{\text {sub }} \rightarrow \mathrm{Te}_{\text {sub }}\right)$ and between the adjacent interior octahedral vacancy and interior tetrahedral vacancy $\left(\mathrm{Oc}_{\text {inte }} \rightarrow \mathrm{Te}_{\text {inte }}\right)$, the reaction coordinates are along the directions that perpendicular to the planes of $\mathrm{Ni}_{1}-\mathrm{Ni}_{2}-$ $\mathrm{Ni}_{3}$ and $\mathrm{Ni}_{4}-\mathrm{Ni}_{5}-\mathrm{Ni}_{6}$, respectively.

3.4.2. Free Energy and Prefactor. In order to investigate the quantized Ni lattice effect on the rates, we have recast the QI formula in (1) into Arrhenius form, which consists of the free energy and the prefactor (17) and (18).

In the free energy calculations, firstly, we calculate the free energy profile for each step of the $\mathrm{H}$ hopping paths, $\mathrm{A}_{\text {hcp }} \rightarrow \mathrm{A}_{\mathrm{fcc}} \rightarrow \mathrm{Oc}_{\text {sub }} \rightarrow \mathrm{Te}_{\text {sub }} \rightarrow \mathrm{A}_{\text {hcp }}$ and $\mathrm{Oc}_{\text {inte }} \rightarrow$ $\mathrm{Te}_{\text {inte }} \rightarrow \mathrm{Oc}_{\text {inte, }}$, with the reaction coordinate defined in Section 3.4.1. Then, we connect these free energy profiles one by one, the final free energy profiles for the whole processes are displayed in Figures 11 and 12.

Figures 11 and 12 display the calculated free energy profiles for the surface-subsurface and interior processes, respectively, with the quantized lattice, the classical lattice and the rigid one at room temperature $(300 \mathrm{~K})$. The corresponding free energy barriers, prefactors, and rates are tabulated in Table 5.

Figures 11 and 12 clearly show that the classical lattices always reduce the free energy barriers compared with the rigid lattices, however, the differences between the free energy barriers with the quantized lattices and the ones with the classical lattices are very small. For different processes;

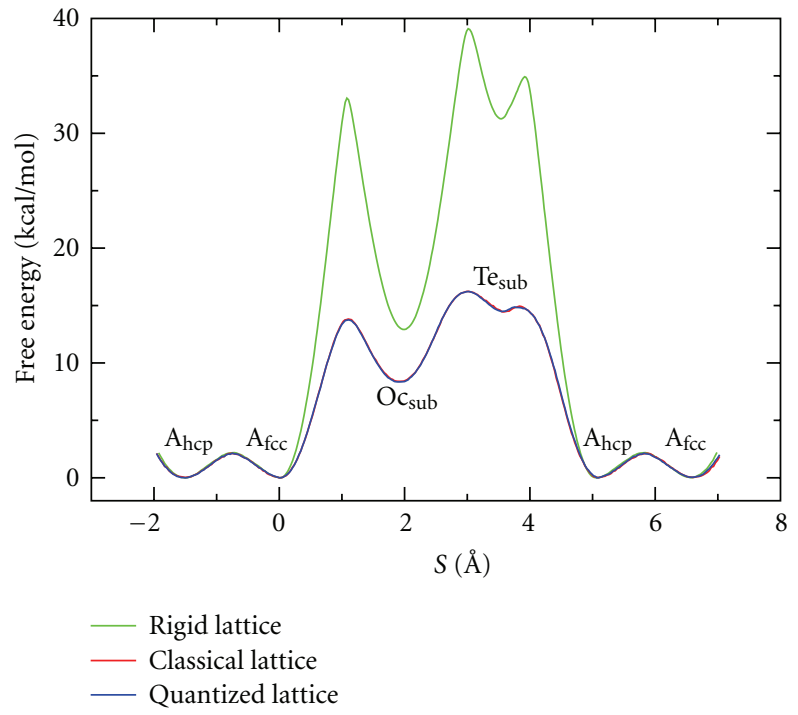

FIgURE 11: Free energy profiles with respect to the processes in Figure 9 at $300 \mathrm{~K}$. The green, the red, and the blue lines correspond to the rigid, classical, and quantized lattices, respectively.

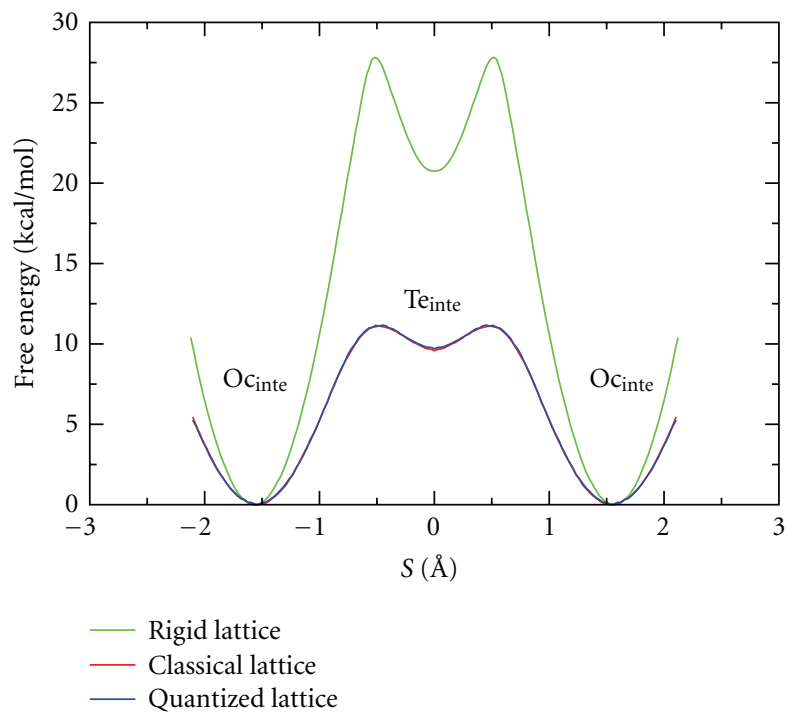

Figure 12: Free energy profiles with respect to the processes in Figure 10 at $300 \mathrm{~K}$. The green, the red, and the blue lines correspond to the rigid, classical, and quantized lattices, respectively.

however, the relaxation effect on the free energies is very different. For hydrogen diffusion on $\mathrm{Ni}(111)$, the classical lattice only slightly lowers the free energy barrier, while it decreases the barriers by more than one-half in subsurface and interior processes. More careful analysis from Figure 11 reveals that the two preferred $\mathrm{Ni}(111)$ surface binding sites, that is, hcp and fcc hollows have symmetric wells, manifesting that the motions of $\mathrm{Ni}$ atoms in the layer beneath the surface have little influence on the surface free energies despite the fact that the $\mathrm{Ni}$ atoms beneath the hcp and fcc hollows have different arrangements. It is also found that the $\mathrm{Oc}_{\text {sub }}$ site has a deeper well than the $\mathrm{Te}_{\text {sub }}$ site and the well of the $\mathrm{Te}_{\text {sub }}$ site nearly disappears as relaxed $\mathrm{Ni}$ atoms 
are considered. It manifests that the hydrogen at the $\mathrm{Te}_{\text {sub }}$ site is very unstable and can easily move to the $\mathrm{Oc}_{\text {sub }}$ site or resurface to the hcp site. In the interior processes, although the hydrogen in the $\mathrm{Te}_{\text {inte }}$ site is much less stable than in the $\mathrm{Oc}_{\text {inte }}$ site (see Figure 12), the well at the $\mathrm{Te}_{\text {inte }}$ is obvious. One thus expects that a two-step reaction process can be used for the reaction from one $\mathrm{Oc}_{\text {inte }}$ to the other.

Next, we consider the prefactor. Table 5 shows that the prefactors of the classical lattices are much smaller than those of rigid ones except for the process of $\mathrm{H}$ diffusion on $\mathrm{Ni}(111)$, and the prefactors of quantized lattices are always smaller than that of classical ones, but their amplitudes have the same order. This may be explained by the fact that the quantum motions of the lattice atoms can induce the dissipative effect on the tunneling degrees of freedom $[62,63]$, because the prefactor essentially incorporates the dynamical effect. It is well known that pure dissipation in the overdamping regime always hinders the reaction rates for a given reaction barrier. The present results thus are consistent with above analysis.

The rate is determined by both the prefactor and the free energy barrier. In Table 5, the rate with the rigid lattice is smaller than the one on the classical lattice, which is because the prefactor changes a little and the free energy determines the rate. However, the rate with the quantized lattice is smaller than that of the classical lattice, which is due to the fact that quantized lattice has a smaller prefactor while the free energies are similar. Generally speaking, for the quantized lattices, the rates are lower by $20 \%-40 \%$ when compared to the ones on the classical lattices.

Another important feature of the free energy is its temperature dependence. Figure 13 displays the free energy profiles with both $\mathrm{H}$ - and $\mathrm{Ni}$-treated quantum mechanically at several temperatures. Generally speaking, the free energies have a slight difference at $300 \mathrm{~K}$ and $400 \mathrm{~K}$, whereas this difference becomes pronounced for $100 \mathrm{~K}$ and $200 \mathrm{~K}$, and the barrier positions move to the directions of shallow well for asymmetric reactions. These properties can be explained by the hydrogen tunneling effect. At lower temperatures, the tunneling plays a more important role. Indeed, the barrier heights decrease with decreasing temperature except for $\mathrm{Oc}_{\text {sub }} \rightarrow \mathrm{Te}_{\text {sub }}$ among $200 \mathrm{~K}$ to $400 \mathrm{~K}$. This special case may be due to the special structure of the lattice. The thermal average displacements of $\mathrm{Ni}_{1}$ and $\mathrm{Ni}_{3}$ (in Figure 9) vertical to the $\mathrm{Ni}(111)$ surface increase with increasing temperature. Thus, $\mathrm{H}$ goes through reaction bottleneck easier at a higher temperature, which makes the barrier decrease. Figure 13 also displays that the free energy barriers of $\mathrm{Oc}_{\text {inte }} \rightarrow \mathrm{Te}_{\text {inte }}$ change little in the temperature range of $200-400 \mathrm{~K}$, while those of the $\mathrm{Te}_{\text {inte }} \rightarrow \mathrm{Oc}_{\text {inte }}$ become smaller and smaller with decreasing temperature. The corresponding free energy barriers are $0.47,1.09,1.47$, and $1.66 \mathrm{kcal} / \mathrm{mol}$ at 100,200 , 300 , and $400 \mathrm{~K}$, respectively. It manifests that the diffusing $\mathrm{H}$ atom may not equilibrate in the interior tetrahedral vacancy at very low temperatures.

3.4.3. Surface-Subsurface Transport. In the resurfacing process, $\mathrm{Te}_{\text {sub }} \rightarrow \mathrm{A}_{\mathrm{hcp}}$ does not show an obvious barrier (see Figure 11), as the lattice atoms are treated quantum mechanically. This step thus can be thought as a barrierless process. Table 6 tabulates the rate constants for the other resurfacing and subsurface processes in the temperature range of 100 to $600 \mathrm{~K}$. We also list the available CVT/SCT results for $\mathrm{Oc}_{\mathrm{sub}} \rightarrow \mathrm{A}_{\mathrm{fcc}}$ and its reverse reaction [60]. Again, the CVT/SCT rates are close to the QI rates except at $100 \mathrm{~K}$. We think our much bigger rate constant at $100 \mathrm{~K}$ is due to the contribution of tunneling.

3.4.4. Interior Migration. In the interior of bulk $\mathrm{Ni}$, the two most stable sites to cage $\mathrm{H}$ are symmetric octahedral vacancies (see Figure 12). H diffusion between them has been measured experimentally [64-67]. Several theoretical calculations have also been proposed to investigate this diffusion process. Wimmer et al. [68] calculate the diffusion coefficient via two-step reactions $\mathrm{Oc}_{\text {inte }} \rightarrow \mathrm{Te}_{\text {inte }} \rightarrow \mathrm{Oc}_{\text {inte }}$ by using a transition state theory together with accurate $\mathrm{ab}$ initio energies, while Wonchoba and Truhlar [60] consider the kinetic step as a direct process with a double maximum barrier and calculate the diffusion coefficient using the CVT/SCT. In the present QI calculations, Figure 13 has explicitly shown that the free energies have a well at the tetrahedral site from $200 \mathrm{~K}$ to $400 \mathrm{~K}$. It is thus reasonable to assume that the diffusing $\mathrm{H}$ atom temporarily equilibrates in the tetrahedral site before jumping forward or backward to a neighboring octahedral site. The free energy well, however, becomes very shallow at $100 \mathrm{~K}$. In this case, the direct reaction from the octahedral site to the other one may be acceptable. Here, we only calculate the diffusion coefficients via the two kinetic steps at $200-400 \mathrm{~K}$.

The temperature dependence of the diffusion coefficients are commonly fitted to the Arrhenius equation

$$
D(T)=D_{0} \cdot \exp \left[\frac{-E_{a}}{R T}\right],
$$

where $R$ is the gas constant and $D_{0}$ and $E_{\mathrm{a}}$ are the preexponential factor and the activation energy, respectively. The QI calculations predict $D_{0}=3.93 \times 10^{-3} \mathrm{~cm}^{2} \mathrm{~s}^{-1}$ and $E_{a}=10.26 \mathrm{kcal} / \mathrm{mol}$. In the calculations, the rates of $\mathrm{Oc}_{\text {inte }} \rightarrow \mathrm{Te}_{\text {inte }}$ are used to obtain the diffusion coefficients for $\mathrm{Oc}_{\text {inte }} \rightarrow \mathrm{Oc}_{\text {inte }}$, because this process is much slower than that of $\mathrm{Te}_{\text {inte }} \rightarrow \mathrm{Oc}_{\text {inte }}$ and it determines the total reaction rates.

Table 7 tabulates the pre-exponential factors and activation energies coming from available experiments and theories. It is found that both $D_{0}$ and $E_{a}$ from the QI calculations are close to Ebisuzaki's experimental data [65]. Further tracking down the comparisons with experiments is nontrivial, because the accuracy of the diffusion coefficients is much dependent of the potential energy surface. However, we may make a quantitative comparison for the QI and CVT/SCT results. With use of the diffusion coefficients from $295 \mathrm{~K}$ to $300 \mathrm{~K}$ obtained by the CVT/SCT, Wonchoba and Truhlar [60] predict $11.1 \mathrm{kcal} / \mathrm{mol}$ for $E_{a}$, and $1.3 \times$ $10^{-3} \mathrm{~cm}^{2} \mathrm{~s}^{-1}$ for $D_{0}$, respectively. These values are observably different from the QI calculations. $E_{a}$ and $D_{0}$ are $0.8 \mathrm{kcal} / \mathrm{mol}$ larger and 3 times smaller than those from the QI calculations, respectively. The origin of these differences can be 
TABle 5: Free energies, prefactors, and rate constants.

\begin{tabular}{|c|c|c|c|c|}
\hline Process $(300 \mathrm{~K})$ & & $\Delta \mathrm{F}(\mathrm{kcal} / \mathrm{mol})$ & $\mathrm{A}_{\mathrm{QI}}\left(\mathrm{s}^{-1}\right)$ & $k_{\mathrm{QI}}\left(\mathrm{s}^{-1}\right)$ \\
\hline \multirow{3}{*}{$\mathrm{A}_{\mathrm{fcc}} \rightarrow \mathrm{A}_{\mathrm{hcp}}$} & $\mathrm{a}$ & 2.19 & $1.58(13)$ & $4.07(11)$ \\
\hline & $\mathrm{b}$ & 2.14 & $1.61(13)$ & $4.49(11)$ \\
\hline & $\mathrm{c}$ & 2.14 & $1.27(13)$ & $3.47(11)$ \\
\hline \multirow{3}{*}{$\mathrm{A}_{\mathrm{fcc}} \rightarrow \mathrm{Oc}_{\mathrm{sub}}$} & $\mathrm{a}$ & 33.06 & $6.87(13)$ & $6.22(-11)$ \\
\hline & $\mathrm{b}$ & 13.79 & $2.85(13)$ & $2.64(3)$ \\
\hline & $\mathrm{c}$ & 13.75 & $2.29(13)$ & $2.23(3)$ \\
\hline \multirow{3}{*}{$\mathrm{Oc}_{\mathrm{sub}} \rightarrow \mathrm{A}_{\mathrm{fcc}}$} & $\mathrm{a}$ & 20.00 & $6.44(13)$ & $1.83(-1)$ \\
\hline & $\mathrm{b}$ & 5.40 & $2.10(13)$ & $2.62(9)$ \\
\hline & $\mathrm{c}$ & 5.40 & $1.72(13)$ & $2.15(9)$ \\
\hline \multirow{3}{*}{$\mathrm{A}_{\mathrm{hcp}} \rightarrow \mathrm{Te}_{\mathrm{sub}}$} & $\mathrm{a}$ & 34.92 & $4.29(13)$ & $1.67(-12)$ \\
\hline & b & 14.92 & & \\
\hline & c & 14.87 & & \\
\hline $\mathrm{Te}_{\text {sub }} \rightarrow \mathrm{A}_{\mathrm{hcp}}$ & $\mathrm{a}$ & 3.25 & $5.13(13)$ & $2.28(11)$ \\
\hline \multirow{3}{*}{$\mathrm{Oc}_{\text {sub }} \rightarrow \mathrm{Te}_{\text {sub }}$} & $\mathrm{a}$ & 26.19 & $5.23(13)$ & $4.31(-6)$ \\
\hline & b & 7.83 & $1.86(13)$ & $3.51(7)$ \\
\hline & c & 7.86 & $1.45(13)$ & $2.74(7)$ \\
\hline \multirow{3}{*}{$\mathrm{Te}_{\mathrm{sub}} \rightarrow \mathrm{Oc}_{\text {sub }}$} & $\mathrm{a}$ & 7.83 & $8.17(13)$ & $1.51(8)$ \\
\hline & b & 1.75 & $1.91(13)$ & $1.05(12)$ \\
\hline & c & 1.73 & $1.49(13)$ & $8.22(11)$ \\
\hline \multirow{3}{*}{$\mathrm{Oc}_{\text {inte }} \rightarrow \mathrm{Te}_{\text {inte }}$} & $\mathrm{a}$ & 27.82 & $5.12(13)$ & $2.86(-7)$ \\
\hline & $\mathrm{b}$ & 11.14 & $2.50(13)$ & $1.90(5)$ \\
\hline & $\mathrm{c}$ & 11.17 & $1.75(13)$ & $1.32(5)$ \\
\hline \multirow{3}{*}{$\mathrm{Te}_{\text {inte }} \rightarrow \mathrm{Oc}_{\text {inte }}$} & $\mathrm{a}$ & 7.07 & $7.80(13)$ & $6.16(8)$ \\
\hline & $\mathrm{b}$ & 1.52 & $2.06(13)$ & $1.60(12)$ \\
\hline & c & 1.47 & $1.53(13)$ & $1.36(12)$ \\
\hline
\end{tabular}

a: The results for a rigid lattice.

b: The results for a classical lattice.

c: The results for a quantized lattice.

TABle 6: Rate constants (Unit: $s^{-1}$. Powers of 10 are in parentheses) for surface-subsurface processes. ${ }^{\text {a }}$ The QI results with a quantized lattice. ${ }^{\mathrm{b}}$ The CVT/SCT results from [60].

\begin{tabular}{|c|c|c|c|c|c|}
\hline & $100 \mathrm{~K}$ & $200 \mathrm{~K}$ & $300 \mathrm{~K}$ & $400 \mathrm{~K}$ & $600 \mathrm{~K}$ \\
\hline $\mathrm{A}_{\mathrm{fcc}} \rightarrow \mathrm{Oc}_{\mathrm{sub}}^{\mathrm{a}}$ & $3.81(-14)$ & $1.14(-1)$ & $2.23(3)$ & $5.48(5)$ & $2.43(8)$ \\
\hline $\mathrm{A}_{\mathrm{fcc}} \rightarrow \mathrm{Oc}_{\mathrm{sub}} \mathrm{b}$ & $1.09(-15)$ & $1.23(-1)$ & $9.72(3)$ & $3.36(6)$ & $1.16(9)$ \\
\hline $\mathrm{Oc}_{\mathrm{sub}} \rightarrow \mathrm{A}_{\mathrm{fcc}}{ }^{\mathrm{a}}$ & $1.17(6)$ & $4.59(7)$ & $2.15(9)$ & $1.23(10)$ & $1.02(11)$ \\
\hline $\mathrm{Oc}_{\mathrm{sub}} \rightarrow \mathrm{A}_{\mathrm{fcc}}^{\mathrm{b}}$ & $1.62(5)$ & $7.36(7)$ & $2.93(9)$ & $2.33(10)$ & $2.15(11)$ \\
\hline $\mathrm{Oc}_{\mathrm{sub}} \rightarrow \mathrm{Te}_{\mathrm{sub}}^{\mathrm{a}}$ & $2.78(-4)$ & $1.99(4)$ & $2.74(7)$ & $1.18(9)$ & $2.49(10)$ \\
\hline $\mathrm{Te}_{\mathrm{sub}} \rightarrow \mathrm{Oc}_{\mathrm{sub}}^{\mathrm{a}}$ & $7.24(8)$ & $4.68(10)$ & $8.22(11)$ & $2.00(12)$ & $4.13(12)$ \\
\hline
\end{tabular}

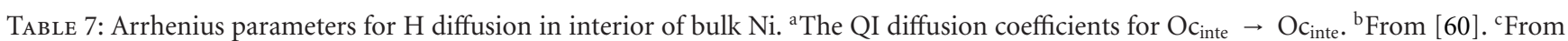
[68]. ${ }^{\mathrm{d}}$ From [64]. ${ }^{\mathrm{e}}$ From [65]. ${ }^{\mathrm{f}}$ From [66]. ${ }^{\mathrm{g}}$ From [67].

\begin{tabular}{|c|c|c|c|}
\hline & & $D_{0}\left(\mathrm{~cm}^{2} \mathrm{~s}^{-1}\right)$ & $E_{a}(\mathrm{kcal} / \mathrm{mol})$ \\
\hline \multirow[t]{2}{*}{ Present QI ${ }^{\mathrm{a}}$} & $200-400 \mathrm{~K}$ & $3.93(-3)$ & 10.26 \\
\hline & $295-300 \mathrm{~K}$ & $1.3(-3)$ & 11.1 \\
\hline \multirow[t]{2}{*}{ Wonchoba ${ }^{\mathrm{b}}$ (theoretical data) } & $300-627 \mathrm{~K}$ & $7.8(-4)$ & 10.9 \\
\hline & $627-1650 \mathrm{~K}$ & $4.4(-4)$ & 10.2 \\
\hline Wimmer ${ }^{c}$ (theoretical data) & $273-1000 \mathrm{~K}$ & $3.84(-2)$ & 10.92 \\
\hline Yamakawa $^{\mathrm{d}}$ (experimental data) & $220-350 \mathrm{~K}$ & $1.9(-3)$ & 8.89 \\
\hline Ebisuzakie (experimental data) & $470-690 \mathrm{~K}$ & $5.22(-3)$ & 9.56 \\
\hline Eichenauer $^{\mathrm{f}}$ (experimental data) & $660-930 \mathrm{~K}$ & $6.73(-3)$ & 9.47 \\
\hline Katz $^{\mathrm{g}}$ (experimental data) & $670-1270 \mathrm{~K}$ & $7.04(-3)$ & 9.43 \\
\hline
\end{tabular}




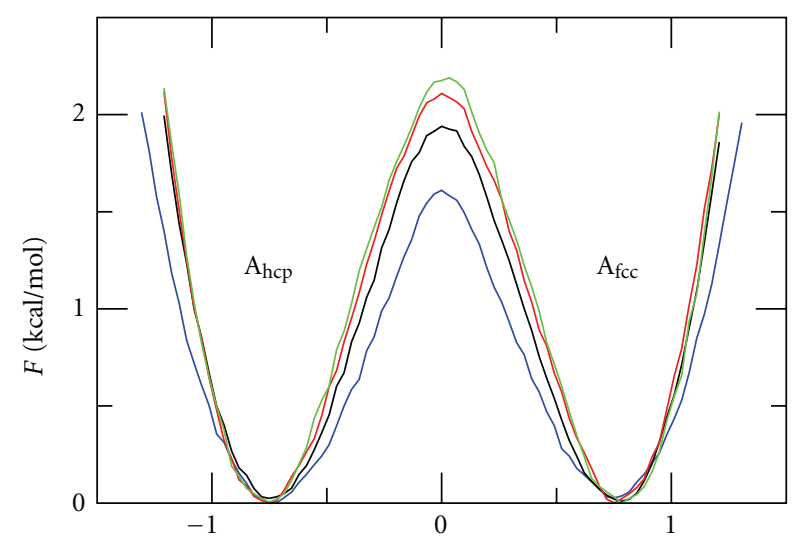

(a)

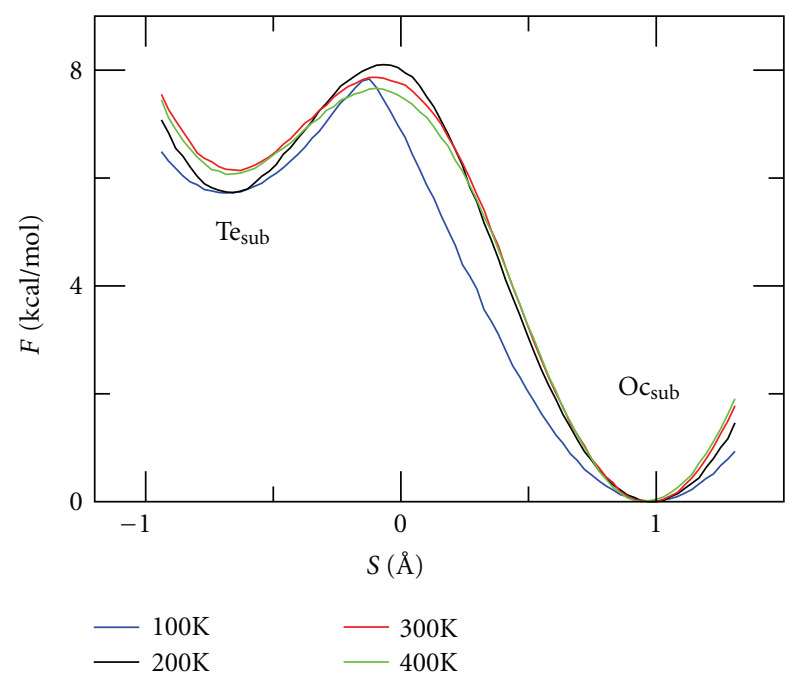

(c)

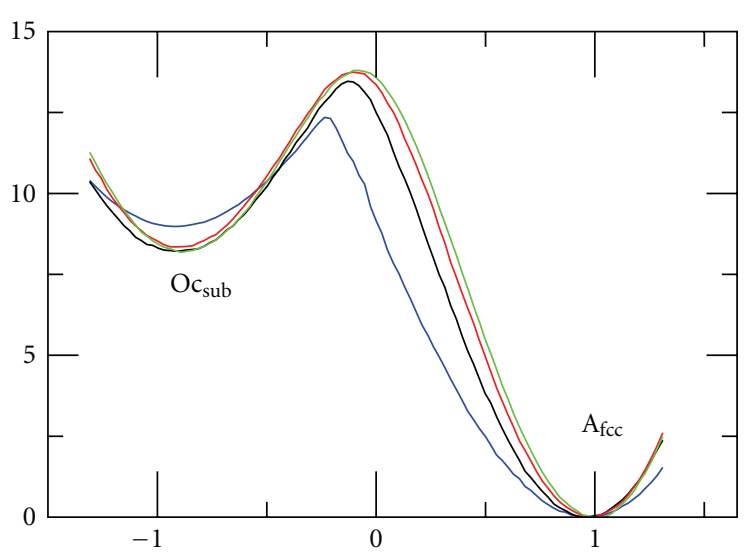

(b)

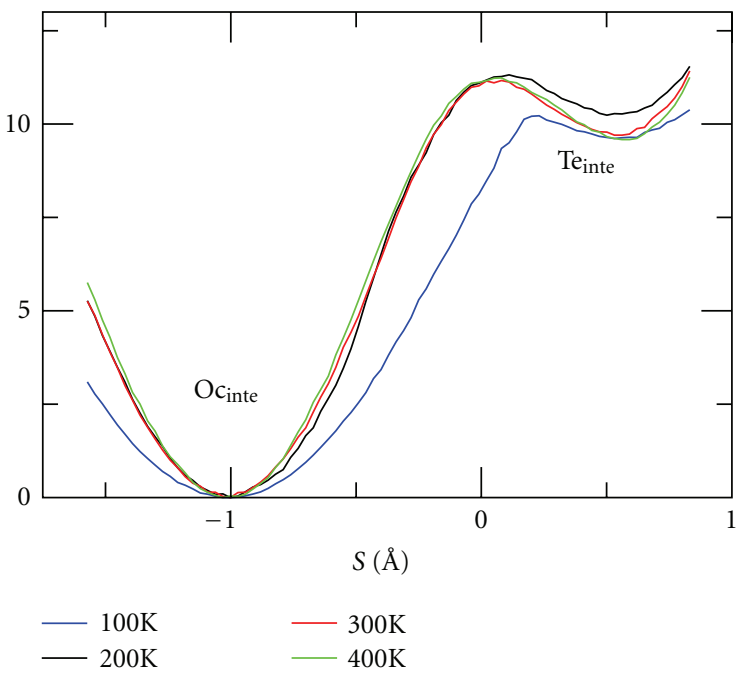

(d)

FIgure 13: The temperature dependence of the free energies. The blue, the black, the red, and the green lines correspond to free energy profiles at $100 \mathrm{~K}, 200 \mathrm{~K}, 300 \mathrm{~K}$, and $400 \mathrm{~K}$, respectively.

explained by that Wonchoba and Truhlar have treated the processes $\mathrm{Oc}_{\text {inte }} \rightarrow \mathrm{Te}_{\text {inte }}$ and $\mathrm{Te}_{\text {inte }} \rightarrow \mathrm{Oc}_{\text {inte }}$ as a single kinetic step rather than as two kinetic steps, which results in a much longer tunneling path than that of the two steps. Compared with the results reported by Wimmer et al. [68], the activation energy is about $0.6 \mathrm{kcal} / \mathrm{mol}$ larger than the present one, and the pre-exponential factor is 10 times larger, which is also larger than all available experimental data. These differences may come from both the different potential energy surfaces and rate methods.

\section{Conclusion}

We have presented the basic principle of the quantum instanton (QI) approximation and its applications to chemical reactions from gas phase to surface systems. The applications demonstrate that the QI method makes it possible to treat hundreds of atoms because of the well-established techniques of imaginary time path integrals. For instance, more than 200 atoms have been incorporated in the present study of the $\mathrm{H}$ diffusion processes.

The QI approximation is a kind of "quantum transition state theory" in that there is no account of "recrossing" dynamics in the description. The recrossing effects on the quantum instanton rate constants have been quantified for several collinear reaction by Ceotto and Miller [25], and it is found especially evident for the collinear heavy-light-heavy reactions. Fortunately, the recrossing effects become generally less important in higher dimensions [69]. Therefore, the QI approach may become a suitable tool for the calculation of chemical reaction rate constants of complex systems.

Compared to conventional TST theories, the QI approximation involves two dividing surfaces, which are quantum analogs of the two turning point surfaces of the imaginary time trajectory in the semiclassical instanton theory. At high-temperature limit, these dividing surfaces coalesce into the one, the same as the dividing surface from Wigner's variational principle. In this case, the QI approximation becomes exactly the same as the classical TST. As the 
tunneling corrections are incorporated, the CVT rates are consistent with the QI rates except at deep tunneling regime, where QI rates are generally greater than the rates from CVT with various tunneling corrections, since the CVT method uses an optimized tunneling path, while the QI method considers all tunneling paths and automatically gives each path its natural weight by the quantum Boltzmann factor. For the reaction of $\mathrm{H}+\mathrm{CH}_{4} \rightarrow \mathrm{H}_{2}+\mathrm{CH}_{3}$, accurate quantum dynamics rate constants are obtained with the MCTDH method. Compared to the MCTDH ones, the QI rate constants are larger by factors of about 2 to 3 over the temperature range $300-400 \mathrm{~K}$. This difference may partly be due to the recrossing effect which is not considered in QI theory and partly arise from the use of the J-shifting approximation and the neglect of the vibrational angular momenta Hamiltonian in MCTDH method.

\section{Acknowledgments}

This work was financially supported by the National Science Foundation of China (Grant nos. 20833004 and 21073146), National Key Basic Research Foundation Program of China (2007CB815204), and the Scientific Research Foundation of Northwest A\&F University (Grant no. Z109021103).

\section{References}

[1] H. Eyring, "The activated complex in chemical reactions," The Journal of Chemical Physics, vol. 3, no. 2, pp. 63-71, 1935.

[2] H. Eyring, "The theory of absolute reaction rates," Transactions of the Faraday Society, vol. 34, pp. 41-48, 1938.

[3] E. Wigner, "The transition state method," Transactions of the Faraday Society, vol. 34, pp. 29-41, 1938.

[4] G. A. Voth, D. Chandler, and W. H. Miller, "Time correlation function and path integral analysis of quantum rate constants," Journal of Physical Chemistry, vol. 93, no. 19, pp. 7009-7015, 1989.

[5] G. A. Voth, D. Chandler, and W. H. Miller, "Rigorous formulation of quantum transition state theory and its dynamical corrections," The Journal of Chemical Physics, vol. 91, no. 12, pp. 7749-7760, 1989.

[6] S. Jang and G. A. Voth, "A relationship between centroid dynamics and path integral quantum transition state theory," Journal of Chemical Physics, vol. 112, no. 20, pp. 8747-8757, 2000.

[7] W. H. Miller, "Generalization of the linearized approximation to the semiclassical initial value representation for reactive flux correlation functions," Journal of Physical Chemistry A, vol. 103, no. 47, pp. 9384-9387, 1999.

[8] E. Pollak and J. L. Liao, "A new quantum transition state theory," Journal of Chemical Physics, vol. 108, no. 7, pp. 27332743, 1998.

[9] J. Shao, J. L. Liao, and E. Pollak, "Quantum transition state theory: perturbation expansion," Journal of Chemical Physics, vol. 108, no. 23, pp. 9711-9725, 1998.

[10] K. Yamashita and W. H. Miller, "'Direct' calculation of quantum mechanical rate constants via path integral methods: application to the reaction path Hamiltonian, with numerical test for the $\mathrm{H}+\mathrm{H}_{2}$ reaction in 3D," The Journal of Chemical Physics, vol. 82, no. 12, pp. 5475-5484, 1984.
[11] J. W. Tromp and W. H. Miller, "New approach to quantum mechanical transition-state theory," Journal of Physical Chemistry, vol. 90, no. 16, pp. 3482-3485, 1986.

[12] N. F. Hansen and H. C. Andersen, "A new formulation of quantum transition state theory for adiabatic rate constants," The Journal of Chemical Physics, vol. 101, no. 7, pp. 6032-6037, 1994.

[13] G. Krilov, E. Sim, and B. J. Berne, "Quantum time correlation functions from complex time Monte Carlo simulations: a maximum entropy approach," Journal of Chemical Physics, vol. 114, no. 3, pp. 1075-1088, 2001.

[14] E. Sim, G. Krilov, and B. J. Berne, "Quantum rate constants from short-time dynamics: an analytic continuation approach," Journal of Physical Chemistry A, vol. 105, no. 12, pp. 2824-2833, 2001.

[15] W. H. Miller, Y. Zhao, M. Ceotto, and S. Yang, "Quantum instanton approximation for thermal rate constants of chemical reactions," Journal of Chemical Physics, vol. 119, no. 3, pp. 1329-1342, 2003.

[16] T. Yamamoto and W. H. Miller, "On the efficient path integral evaluation of thermal rate constants within the quantum instanton approximation," Journal of Chemical Physics, vol. 120, no. 7, pp. 3086-3099, 2004.

[17] Y. Zhao, T. Yamamoto, and W. H. Miller, "Path integral calculation of thermal rate constants within the quantum instanton approximation: application to the $\mathrm{H}+\mathrm{CH}_{4} \rightarrow \mathrm{H}_{2}+$ $\mathrm{CH}_{3}$ hydrogen abstraction reaction in full Cartesian space," Journal of Chemical Physics, vol. 120, no. 7, pp. 3100-3107, 2004.

[18] W. H. Miller, "Semiclassical limit of quantum mechanical transition state theory for nonseparable systems," The Journal of Chemical Physics, vol. 62, no. 5, pp. 1899-1906, 1975.

[19] S. Coleman, "Fate of the false vacuum: semiclassical theory," Physical Review D, vol. 15, no. 10, pp. 2929-2936, 1977.

[20] A. J. Leggett, S. Chakravarty, A. T. Dorsey, M. P. A. Fisher, A. Garg, and W. Zwerger, "Dynamics of the dissipative two-state system," Reviews of Modern Physics, vol. 59, no. 1, pp. 1-85, 1987.

[21] R. A. Marcus, "On the analytical mechanics of chemical reactions. quantum mechanics of linear collisions," The Journal of Chemical Physics, vol. 45, no. 12, pp. 4493-4499, 1966.

[22] E. A. Mccullough and R. E. Wyatt, "Dynamics of the collinear $\mathrm{H}+\mathrm{H}_{2}$ reaction. I. Probability density and flux," The Journal of Chemical Physics, vol. 54, no. 8, pp. 3578-3591, 1971.

[23] D. G. Truhlar and A. Koppermann, "Exact and approximate quantum mechanical reaction probabilities and rate constants for the collinear $\mathrm{H}+\mathrm{H}_{2}$ reaction," The Journal of Chemical Physics, vol. 56, no. 5, pp. 2232-2252, 1972.

[24] T. V. George and W. H. Miller, "Classical S-matrix theory of reactive tunneling: linear $\mathrm{H}+\mathrm{H}_{2}$ collisions," The Journal of Chemical Physics, vol. 57, no. 6, pp. 2458-2467, 1972.

[25] M. Ceotto and W. H. Miller, "Test of the quantum instanton approximation for thermal rate constants for some collinear reactions," Journal of Chemical Physics, vol. 120, no. 14, pp. 6356-6362, 2004.

[26] C. Venkataraman and W. H. Miller, "The Quantum Instanton (QI) model for chemical reaction rates: the "simplest" QI with one dividing surface," Journal of Physical Chemistry A, vol. 108, no. 15, pp. 3035-3039, 2004.

[27] Y. Li and W. H. Miller, "Different time slices for different degrees of freedom in Feynman path integration," Molecular Physics, vol. 103, no. 2-3, pp. 203-208, 2005.

[28] T. Yamamoto and W. H. Miller, "Path integral evaluation of the quantum instanton rate constant for proton transfer in a polar 
solvent," Journal of Chemical Physics, vol. 122, no. 4, Article ID 044106, pp. 1-13, 2005.

[29] M. Ceotto, S. Yang, and W. H. Miller, "Quantum reaction rate from higher derivatives of the thermal flux-flux autocorrelation function at time zero," Journal of Chemical Physics, vol. 122, no. 4, Article ID 044109, pp. 1-7, 2005.

[30] J. Vaníček, W. H. Miller, J. F. Castillo, and F. J. Aoiz, "Quantum-instanton evaluation of the kinetic isotope effects," Journal of Chemical Physics, vol. 123, no. 5, Article ID 054108, pp. 1-14, 2005.

[31] Y. Li and W. H. Miller, "Using a family of dividing surfaces normal to the minimum energy path for quantum instanton rate constants," Journal of Chemical Physics, vol. 125, no. 6, Article ID 064104, 2006.

[32] S. Yang, T. Yamamoto, and W. H. Miller, "Path-integral virial estimator for reaction-rate calculation based on the quantum instanton approximation," Journal of Chemical Physics, vol. 124, no. 8, Article ID 084102, 2006.

[33] J. Vaníček and W. H. Miller, "Efficient estimators for quantum instanton evaluation of the kinetic isotope effects: application to the intramolecular hydrogen transfer in pentadiene," Journal of Chemical Physics, vol. 127, no. 11, Article ID 114309, 2007.

[34] M. Buchowiecki and J. Vaníček, "Direct evaluation of the temperature dependence of the rate constant based on the quantum instanton approximation," Journal of Chemical Physics, vol. 132, no. 19, Article ID 194106, 2010.

[35] T. Zimmermann and J. Vaníček, "Three applications of path integrals: equilibrium and kinetic isotope effects, and the temperature dependence of the rate constant of the $[1,5]$ sigmatropic hydrogen shift in (Z)-1,3-pentadiene," Journal of Molecular Modeling, vol. 16, no. 11, pp. 1779-1787, 2010.

[36] K. F. Wong, J. L. Sonnenberg, F. Paesani et al., "Proton transfer studied using a combined ab initio reactive potential energy surface with quantum path integral methodology," Journal of Chemical Theory and Computation, vol. 6, no. 9, pp. 25662580, 2010.

[37] W. Wang, S. Feng, and Y. Zhao, "Quantum instanton evaluation of the thermal rate constants and kinetic isotope effects for $\mathrm{SiH}_{4}+\mathrm{H} \rightarrow \mathrm{SiH}_{3}+\mathrm{H}_{2}$ reaction in full Cartesian space," Journal of Chemical Physics, vol. 126, no. 11, Article ID 114307, 2007.

[38] W. Wang and Y. Zhao, "Path integral evaluation of $\mathrm{H}$ diffusion on $\mathrm{Ni}(100)$ surface based on the quantum instanton approximation," Journal of Chemical Physics, vol. 130, no. 11, Article ID 114708, pp. 1-10, 2009.

[39] W. Wang and Y. Zhao, "Quantum instanton evaluations of surface diffusion, interior migration, and surface-subsurface transport for H/Ni," Journal of Chemical Physics, vol. 132, no. 6, Article ID 064502, 2010.

[40] B. J. Berne and D. Thirumalai, "On the simulation of quantum systems: path integral methods," Annual Review of Physical Chemistry, vol. 37, pp. 401-424, 1986.

[41] R. P. Feynman and A. R. Hibbs, Quantum Mechanics and Path Integrals, McGraw-Hill, New York, NY, USA, 1965.

[42] R. P. Feynman, Statistical Mechanics, Addison-Wesley, New York, NY, USA, 1972.

[43] L. S. Schulman, Techniques and Applications of Path Integrals, John Wiley \& Sons, New York, NY, USA, 1986.

[44] M. Mezei, "Adaptive umbrella sampling: self-consistent determination of the non-Boltzmann bias," Journal of Computational Physics, vol. 68, no. 1, pp. 237-248, 1987.
[45] C. Predescu and W. H. Miller, "Optimal choice of dividing surface for the computation of quantum reaction rates," Journal of Physical Chemistry B, vol. 109, no. 14, pp. 64916499, 2005.

[46] J. Espinosa-García, "New analytical potential energy surface for the $\mathrm{CH}_{4}+\mathrm{H}$ hydrogen abstraction reaction: thermal rate constants and kinetic isotope effects," Journal of Chemical Physics, vol. 116, no. 24, pp. 10664-10673, 2002.

[47] D. L. Baulch, C. J. Cobos, R. A. Cox et al., "Evaluated kinetic data for combustion modelling," Journal of Physical and Chemical Reference Data, vol. 21, no. 3, pp. 411-734, 1992.

[48] J. W. Sutherland, M. C. Su, and J. V. Michael, "Rate constants for $\mathrm{H}+\mathrm{CH}_{4}, \mathrm{CH}_{3}+\mathrm{H}_{2}$, and $\mathrm{CH}_{4}$ dissociation at high temperature," International Journal of Chemical Kinetics, vol. 33, no. 11, pp. 669-684, 2001.

[49] S. Andersson, G. Nyman, A. Arnaldsson, U. Manthe, and H. Jonsson, "Comparison of quantum dynamics and quantum transition state theory estimates of the $\mathrm{H}+\mathrm{CH}_{4}$ reaction rate," Journal of Physical Chemistry A, vol. 113, no. 16, pp. 44684478, 2009.

[50] J. Espinosa-García, J. Sansón, and J. C. Corchado, "The $\mathrm{SiH}_{4}+$ $\mathrm{H} \rightarrow \mathrm{SiH}_{3}+\mathrm{H}_{2}$ reaction: potential energy surface, rate constants, and kinetic isotope effects," Journal of Chemical Physics, vol. 109, no. 2, pp. 466-473, 1998.

[51] K. D. Dobbs and D. A. Dixon, "Ab initio prediction of the activation energies for the abstraction and exchange reactions of $\mathrm{H}$ with $\mathrm{CH}_{4}$ and $\mathrm{SiH}_{4}$," Journal of Physical Chemistry, vol. 98, no. 20, pp. 5290-5297, 1994.

[52] A. Goumri, W. J. Yuan, L. Ding, Y. Shi, and P. Marshall, "Experimental and theoretical studies of the reaction of atomic hydrogen with silane," Chemical Physics, vol. 177, no. 1, pp. 233-241, 1993.

[53] T. N. Truong and D. G. Truhlar, "Effect of steps and surface coverage on rates and kinetic isotope effects for reactions catalyzed by metallic surfaces: chemisorption of hydrogen on Ni," Journal of Physical Chemistry, vol. 94, no. 21, pp. 82628279, 1990.

[54] J. V. Barth, "Transport of adsorbates at metal surfaces: from thermal migration to hot precursors," Surface Science Reports, vol. 40, no. 3, pp. 75-149, 2000.

[55] T. N. Truong and D. G. Truhlar, "The effects of steps, coupling to substrate vibrations, and surface coverage on surface diffusion rates and kinetic isotope effects: hydrogen diffusion on Ni," The Journal of Chemical Physics, vol. 93, no. 3, pp. 2125-2138, 1990.

[56] S. M. George, A. M. DeSantolo, and R. B. Hall, "Surface diffusion of hydrogen on $\mathrm{Ni}(100)$ studied using laser-induced thermal desorption," Surface Science, vol. 159, no. 1, pp. L425L432, 1985.

[57] D. R. Mullins, B. Roop, S. A. Costello, and J. M. White, "Isotope effects in surface diffusion: hydrogen and deuterium on Ni(100)," Surface Science, vol. 186, no. 1-2, pp. 67-74, 1987.

[58] A. Lee, X. D. Zhu, L. Deng, and U. Linke, "Observation of a transition from over-barrier hopping to activated tunneling diffusion: $\mathrm{H}$ and $\mathrm{D}$ on $\mathrm{Ni}(100)$," Physical Review B, vol. 46, no. 23, pp. 15472-15476, 1992.

[59] T. S. Lin and R. Gomer, "Diffusion of ${ }^{1} \mathrm{H}$ and ${ }^{2} \mathrm{H}$ on the $\mathrm{Ni}(111)$ and (100) planes," Surface Science, vol. 255, no. 1-2, pp. 41-60, 1991.

[60] S. E. Wonchoba and D. G. Truhlar, "General potentialenergy function for $\mathrm{H} / \mathrm{Ni}$ and dynamics calculations of surface diffusion, bulk diffusion, subsurface-to-surface transport, and 
absorption," Physical Review B, vol. 53, no. 16, pp. 1122211241, 1996.

[61] T. N. Truong, D. G. Truhlar, and B. C. Garrett, "Embedded diatomics-in-molecules: a method to include delocalized electronic interactions in the treatment of covalent chemical reactions at metal surfaces," Journal of Physical Chemistry, vol. 93, no. 25, pp. 8227-8239, 1989.

[62] R. Baer and R. Kosloff, "Quantum dissipative dynamics of adsorbates near metal surfaces: a surrogate Hamiltonian theory applied to hydrogen on nickel," Journal of Chemical Physics, vol. 106, no. 21, pp. 8862-8875, 1997.

[63] R. Baer, Y. Zeiri, and R. Kosloff, "Hydrogen transport in nickel (111)," Physical Review B, vol. 55, no. 16, pp. 10952-10974, 1997.

[64] K. Yamakawa, "Diffusion of deuterium and isotope effect in nickel," Journal of the Physical Society of Japan, vol. 47, no. 1, pp. 114-121, 1979.

[65] Y. Ebisuzaki, W. J. Kass, and M. O'Keeffe, "Diffusion and solubility of hydrogen in single crystals of nickel and nickelvanadium alloy," The Journal of Chemical Physics, vol. 46, no. 4, pp. 1378-1381, 1967.

[66] W. Eichenauer, W. Loser, and H. Witte, "Solubility and rate of diffusion of hydrogen and deuterium in nickel and copper single crystals," Z Metallk, vol. 56, pp. 287-293, 1965.

[67] L. Katz, M. Guinan, and R. J. Borg, "Diffusion of $\mathrm{H}_{2}, \mathrm{D}_{2}$, and $\mathrm{T}_{2}$ in single-crystal $\mathrm{Ni}$ and Cu," Physical Review B, vol. 4, no. 2, pp. 330-341, 1971.

[68] E. Wimmer, W. Wolf, J. Sticht et al., “Temperature-dependent diffusion coefficients from ab initio computations: hydrogen, deuterium, and tritium in nickel," Physical Review B, vol. 77, no. 13, Article ID 134305, 2008.

[69] S. Chapman, S. M. Hornstein, and W. H. Miller, "Accuracy of transition state theory for the threshold of chemical reactions with activation energy. Collinear and three-dimensional $\mathrm{H}+$ $\mathrm{H}_{2}$," Journal of the American Chemical Society, vol. 97, no. 4, pp. 892-894, 1975. 


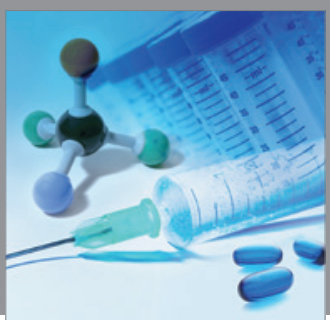

International Journal of

Medicinal Chemistry

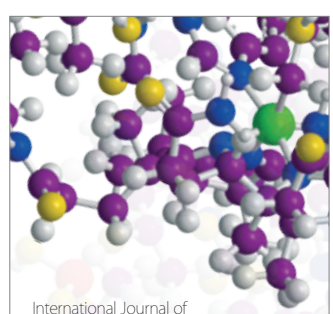

Carbohydrate Chemistry

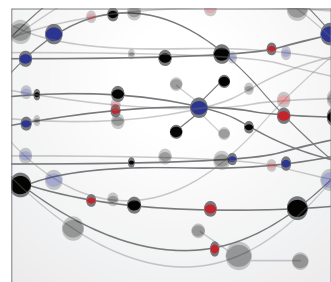

The Scientific World Journal
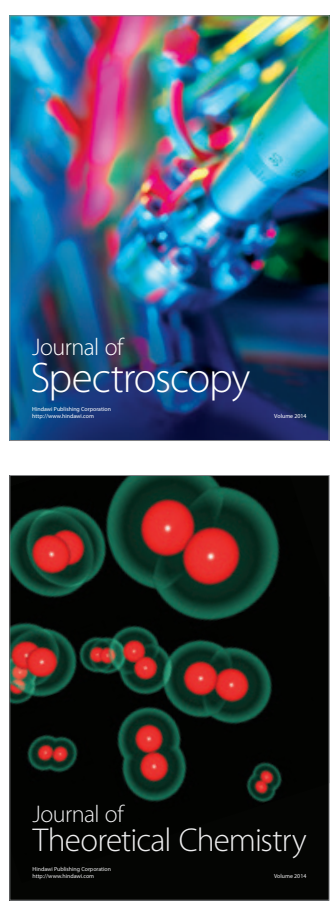
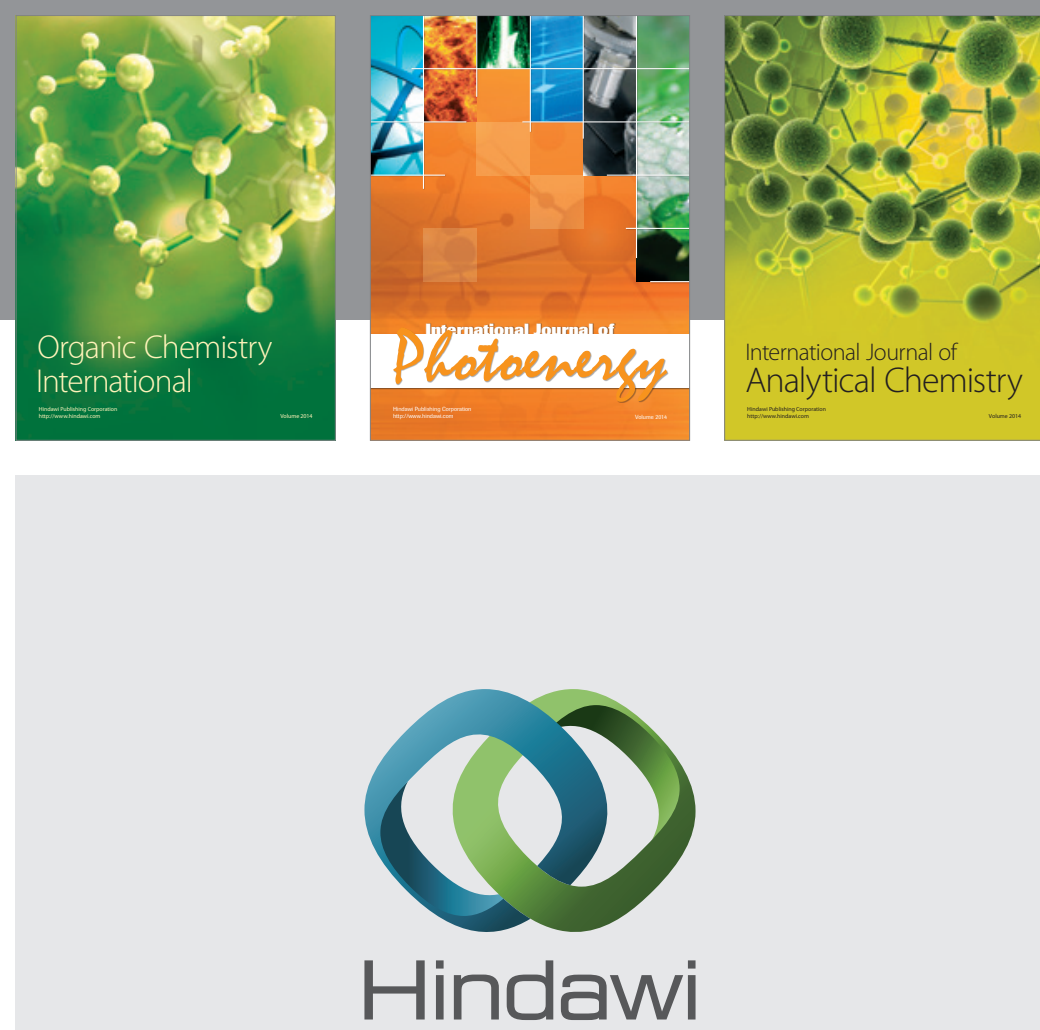

Submit your manuscripts at

http://www.hindawi.com
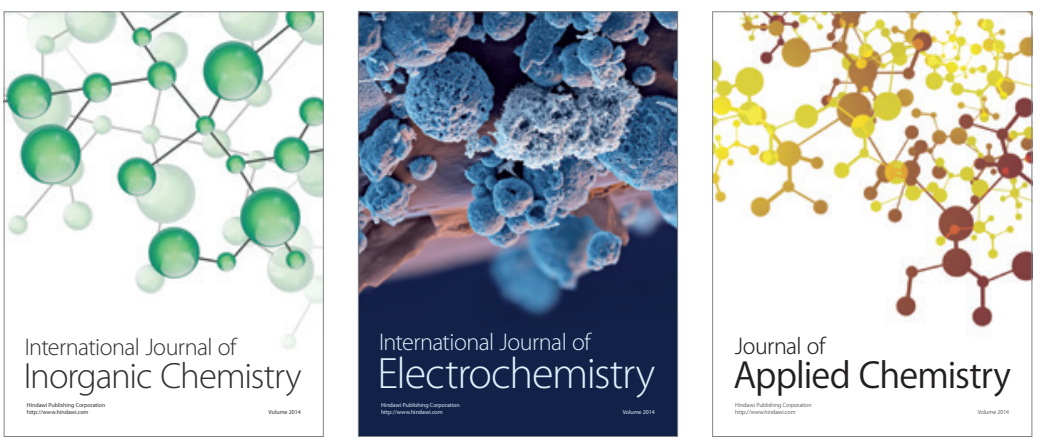

Journal of

Applied Chemistry
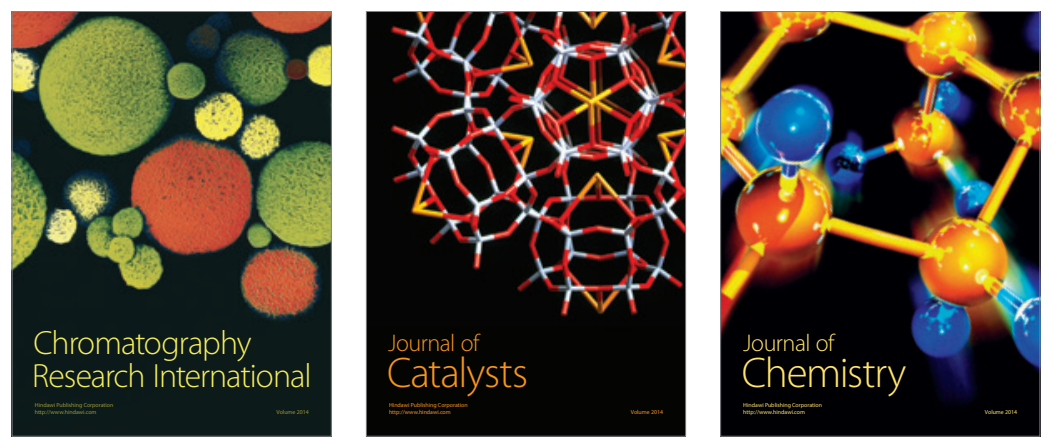
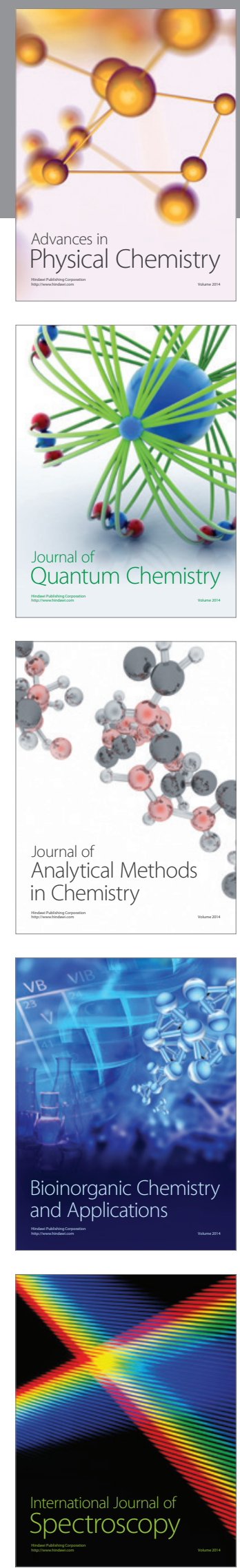